Supporting Information Suhre, Reif, Kirsch

\title{
Gold(I)-Catalyzed Synthesis of Highly Substituted Furans
}

\author{
Michael H. Suhre, Michael Reif, Stefan F. Kirsch* \\ Lehrstuhl für Organische Chemie I, Technische Universität München, Lichtenbergstr. 4, D- \\ 85747 Garching, Germany
}

\section{Supporting Information}

Representative experimental procedures, copies of ${ }^{1} \mathrm{H}$ NMR spectra of $\mathbf{3 a}-\mathbf{q}$, and copies of ${ }^{13} \mathrm{C}$ NMR spectra of $\mathbf{3 a}-\mathbf{q}$.

\section{(23 Pages)}

General experimental details: All reactions were performed under Argon. All propargyl vinyl ethers 1 were synthesized by the reaction of the corresponding propargylic alcohols with 2-propynoic acid derivatives in the presence of trimethylphosphine. ${ }^{1}$ 3-Butin-1-ol was purchased from Acros; and ethyl 2butynoate, methyl 2-octynoate, ethyl propiolate, ethyl phenylpropiolate and 3-pentyn-2-ol were purchased from Aldrich. All commercially available chemicals were used without further purification. All the other propargylic alcohols were prepared from the corresponding alkynes by addition to paraformaldehyde. ${ }^{2}$

${ }^{1} \mathrm{H}$ NMR spectra were obtained on Bruker $500 \mathrm{MHz}$ FT-NMR and $360 \mathrm{MHz}$ FT-NMR spectrometers. ${ }^{13} \mathrm{C}$ NMR spectra were recorded at 90.6 MHz. Chemical shifts are reported in ppm relative to solvent signal. Multiplicity is indicated as follows: s (singlet); d (doublet); t (triplet); q (quartet); m (multiplet); dd (doublet of doublets); etc.; br (broad); app (apparent). High resolution mass spectra and EI were determined on a Finnigan MAT 95S and MAT 8200. Flash chromatography was performed with E. Merck silica gel $(43-60 \mu \mathrm{m})$. The eluent used is reported in parentheses. Thin-layer chromatography (TLC) was performed on precoated glass-backed plates (Merck Kieselgel $60 \mathrm{~F}_{254}$ ), and components were visualized by observation under UV light or by treating the plates with $\mathrm{KMnO}_{4} / \mathrm{H}_{2} \mathrm{SO}_{4}$ followed by heating. For the addition of $\left(\mathrm{PPh}_{3}\right) \mathrm{AuCl}$ to the reaction mixture, a stock solution was used.

\footnotetext{
${ }^{1}$ Inanaga, J.; Baba, Y.; Hanamoto, T. Chem. Lett. 1993, 241-244.

${ }^{2}$ Hashmi, A. S. K.; Haufe, P.; Nass, A. R. Adv. Synth. Catal. 2003, 345, 1237-1241.
} 


\section{General Procedure for the Furan Formation Catalyzed by Gold(I).}

Ethyl 2,4,5-trimethylfuran-3-carboxylate (3a). ${ }^{3}\left(\mathrm{PPh}_{3}\right) \mathrm{AuCl}(2 \mathrm{~mol} \%, 2.2 \mathrm{mg})$ and $\mathrm{AgBF}_{4}(2$ mol\%, $0.9 \mathrm{mg}$ ) were added subsequently to a solution of $1 \mathbf{a}(40.0 \mathrm{mg}, 0.22 \mathrm{mmol})$ in $\mathrm{CH}_{2} \mathrm{Cl}_{2}(1.1$ $\mathrm{mL}$ ), and the reaction vial was sealed, protected from light, and stirred at room temperature. The dark reaction mixture was stirred at room temperature for $4 \mathrm{~h}$ (until DC analysis indicated complete conversion). The mixture was concentrated under reduced pressure. Purification of the residue by flash chromatography on silica gel $(\mathrm{P} / \mathrm{EtOAc}=95 / 5)$ gave furan $3 \mathbf{a}$ as a colorless oil (38.0 mg, $0.21 \mathrm{mmol}, 95 \%): \mathrm{R}_{\mathrm{f}} 0.71$ (20\% EtOAc/hexanes). ${ }^{1} \mathrm{H}$ NMR (360 MHz, $\mathrm{CDCl}_{3}$ ) $\delta 1.34$ (t, $J=7.3 \mathrm{~Hz}, 3 \mathrm{H}), 2.05$ (s, $3 \mathrm{H}), 2.16$ (s, $3 \mathrm{H}), 2.49$ (s, $3 \mathrm{H}), 4.26$ (q, $J=7.3 \mathrm{~Hz}, 2 \mathrm{H})$; ${ }^{13} \mathrm{C}$ NMR

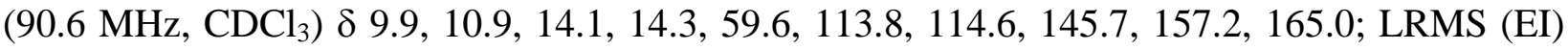
182 (60\%) [ $\mathrm{M}^{+}$], 153 (78\%) [ $\left.\mathrm{M}^{+}-\mathrm{CH}_{2} \mathrm{CH}_{3}\right], 43$ (100\%); HRMS 182.0945 [182.0943 calcd for $\left.\mathrm{C}_{10} \mathrm{H}_{14} \mathrm{O}_{3}^{+}\right]$.

Methyl 4,5-dimethyl-2-pentylfuran-3-carboxylate (3b). Following the general procedure, $\mathbf{3 b}$ (58.2 mg, $0.26 \mathrm{mmol}$, 97\%) was obtained as a pale yellow oil from $\mathbf{1 b}$ (60.0 mg, $0.27 \mathrm{mmol})$. The reaction was conducted in $\mathrm{CH}_{2} \mathrm{Cl}_{2}(1.35 \mathrm{~mL})$ in the presence of $\left(\mathrm{PPh}_{3}\right) \mathrm{AuCl}(2$ mol\%, $2.7 \mathrm{mg}$ ) and $\mathrm{AgBF}_{4}(2 \mathrm{~mol} \%, 1.1 \mathrm{mg})$ at $23{ }^{\circ} \mathrm{C}$ for $15 \mathrm{~h}$. The residue was purified by flash chromatography on silica (3\% EtOAc/pentane): $\mathrm{R}_{\mathrm{f}} 0.73$ (20\% EtOAc/hexanes). ${ }^{1} \mathrm{H}$ NMR (360 $\left.\mathrm{MHz}, \mathrm{CDCl}_{3}\right) \delta 0.89(\mathrm{t}, J=6.8 \mathrm{~Hz}, 3 \mathrm{H}), 1.25-1.34(\mathrm{~m}, 4 \mathrm{H}), 1.55-1.62$ (m, $\left.2 \mathrm{H}\right), 2.05$ (s, $\left.3 \mathrm{H}\right)$, 2.16 (s, $3 \mathrm{H}$ ), 2.88 (t, $J=7.7 \mathrm{~Hz}, 2 \mathrm{H}), 3.79$ (s, $3 \mathrm{H}) ;{ }^{13} \mathrm{C}$ NMR (90.6 MHz, $\mathrm{CDCl}_{3}$ ) $\delta$ 9.9, 11.0, 13.9, 22.4, 27.9, 28.0, 31.4, 50.8, 113.1, 114.4, 145.8, 161.5, 165.4; LRMS (EI) 224 (30\%) [M ${ }^{+}$, 167 (100\%), 43 (28\%); HRMS 224.1413 [224.1412 calcd for $\mathrm{C}_{13} \mathrm{H}_{20} \mathrm{O}_{3}{ }^{+}$].

Ethyl 4,5-dimethyl-2-phenylfuran-3-carboxylate (3c). Following the general procedure, 3c (45.1 mg, $0.18 \mathrm{mmol}, 90 \%$ ) was obtained as a colorless oil from 1c (50.0 mg, $0.20 \mathrm{mmol}$ ). The reaction was conducted in $\mathrm{CH}_{2} \mathrm{Cl}_{2}(1.00 \mathrm{~mL})$ in the presence of $\left(\mathrm{PPh}_{3}\right) \mathrm{AuCl}(2 \mathrm{~mol} \%, 2.0 \mathrm{mg})$ and $\mathrm{AgBF}_{4}(2 \mathrm{~mol} \%, 0.8 \mathrm{mg})$ at $23{ }^{\circ} \mathrm{C}$ for $2 \mathrm{~h}$. The residue was purified by flash chromatography on silica (2\% EtOAc/pentane): $\mathrm{R}_{\mathrm{f}} 0.63$ (20\% EtOAc/hexanes). ${ }^{1} \mathrm{H}$ NMR (500 MHz, $\left.\mathrm{CDCl}_{3}\right) \delta$ 1.29 (t, $J=7.1 \mathrm{~Hz}, 3 \mathrm{H}), 2.12$ (s, $3 \mathrm{H}), 2.28$ (s, $3 \mathrm{H}), 4.27$ (q, $J=7.1 \mathrm{~Hz}, 2 \mathrm{H}), 7.30-7.41$ (m, 3

\footnotetext{
${ }^{3}$ Minami, I.; Yuhara, M.; Watanabe, H.; Tsuji, J. J. Organomet. Chem. 1987, 334, 225-242.
} 
H), 7.72-7.79 (m, $2 \mathrm{H}) ;{ }^{13} \mathrm{C}$ NMR (90.6 MHz, $\left.\mathrm{CDCl}_{3}\right) \delta$ 9.9, 11.3, 14.1, 60.2, 114.7, 116.3, 127.9, 128.2, 128.6, 130.6, 147.6, 155.1, 164.8; LRMS (EI) 244 (100\%) $\left[\mathrm{M}^{+}\right], 216$ (30\%) $\left[\mathrm{M}^{+}-\mathrm{C}_{2} \mathrm{H}_{4}\right], 199$ (42\%); HRMS 244.1098 [244.1099 calcd for $\left.\mathrm{C}_{15} \mathrm{H}_{16} \mathrm{O}_{3}{ }^{+}\right]$.

Ethyl 4,5-dimethylfuran-3-carboxylate (3d). Following the general procedure, 3d (47.9 mg, $0.28 \mathrm{mmol}, 75 \%)$ was obtained as a yellow oil from 1d $(63.9 \mathrm{mg}, 0.38 \mathrm{mmol})$ which was contaminated with an unknown side product $(\sim 3 \%)$. The reaction was conducted in benzene (1.90 $\mathrm{mL}$ ) in the presence of $\left(\mathrm{PPh}_{3}\right) \mathrm{AuCl}(2 \mathrm{~mol} \%, 3.8 \mathrm{mg})$ and $\mathrm{AgBF}_{4}(2 \mathrm{~mol} \%, 1.5 \mathrm{mg})$ at $23{ }^{\circ} \mathrm{C}$ for 4 $h$. The residue was purified by flash chromatography on silica (10\% Et $\mathrm{t}_{2} \mathrm{O} /$ pentane): $\mathrm{R}_{\mathrm{f}} 0.71$ ( $20 \%$ EtOAc/hexanes). ${ }^{1} \mathrm{H}$ NMR (360 MHz, $\mathrm{CDCl}_{3}$ ) $\delta 1.33$ (t, $J=7.0 \mathrm{~Hz}, 3 \mathrm{H}$ ), 2.10 (s, $3 \mathrm{H}$ ), 2.21 (s, 3 H), 4.27 (q, $J=7.0 \mathrm{~Hz}, 2 \mathrm{H}), 7.82(\mathrm{~s}, 1 \mathrm{H}) ;{ }^{13} \mathrm{C} \mathrm{NMR}\left(90.6 \mathrm{MHz}, \mathrm{CDCl}_{3}\right) \delta$ 9.0, 11.2, 14.3, 59.9, 114.3, 119.3, 146.0, 149.4, 164.1; LRMS (EI) 168 (55\%) [ $\left.\mathrm{M}^{+}\right], 139$ (100\%) $\left[\mathrm{M}^{+}-\mathrm{CH}_{2} \mathrm{CH}_{3}\right], 123$ (60\%), 43 (62\%); HRMS 168.0788 [168.0786 calcd for $\mathrm{C}_{9} \mathrm{H}_{12} \mathrm{O}_{3}{ }^{+}$].

(4,5-dimethyl-2-phenylfuran-3-yl)(phenyl)methanone (3e). Following the general procedure, 3e (35.8 mg, $0.13 \mathrm{mmol}, 72 \%$ ) was obtained as a colorless oil from 1 e (50.0 mg, $0.18 \mathrm{mmol}$ ). The reaction was conducted in $\mathrm{CH}_{2} \mathrm{Cl}_{2}(0.9 \mathrm{~mL})$ in the presence of $\left(\mathrm{PPh}_{3}\right) \mathrm{AuCl}(2 \mathrm{~mol} \%, 1.8 \mathrm{mg})$ and $\mathrm{AgBF}_{4}\left(2 \mathrm{~mol} \%, 0.7 \mathrm{mg}\right.$ ) at $23^{\circ} \mathrm{C}$ for $8 \mathrm{~h}$. The residue was purified by flash chromatography on silica (2\% EtOAc/pentane): $\mathrm{R}_{\mathrm{f}} 0.59$ (20\% EtOAc/hexanes). ${ }^{1} \mathrm{H}$ NMR (360 MHz, $\mathrm{CDCl}_{3}$ ) $\delta 1.91$ (s, 3 H), 2.33 (s, 3 H), 7.05-7.22 (m, 3 H), 7.28-7.40 (m, 2 H), 7.42-7.51 (m, 3 H), 7.81-7.84 (m, $2 \mathrm{H}) ;{ }^{13} \mathrm{C}$ NMR $\left(90.6 \mathrm{MHz}, \mathrm{CDCl}_{3}\right) \delta 8.9,11.4,116.4,122.3,126.6,127.9,128.2,128.3$, 129.8, 130.1, 133.0, 137.9, 147.8, 151.5, 193.9; LRMS (EI) 276 (100\%) $\left[\mathrm{M}^{+}\right], 199$ (30\%) $\left[\mathrm{M}^{+}-\mathrm{C}_{6} \mathrm{H}_{5}\right.$ ], 105 (82\%), 77 (91\%) $\left[\mathrm{C}_{6} \mathrm{H}_{5}{ }^{+}\right.$]; HRMS 276.1148 [276.1150 calcd for $\mathrm{C}_{19} \mathrm{H}_{16} \mathrm{O}_{2}{ }^{+}$].

Ethyl 2,5-dimethylfuran-3-carboxylate (3f) ${ }^{4}$ Following the general procedure, $3 \mathbf{3 f}$ (41.1 $\mathrm{mg}$, $0.24 \mathrm{mmol}, 82 \%)$ was obtained as a pale yellow oil from $1 \mathbf{1 f}(50.0 \mathrm{mg}, 0.29 \mathrm{mmol})$. The reaction was conducted in $\mathrm{CH}_{2} \mathrm{Cl}_{2}(1.45 \mathrm{~mL})$ in the presence of $\left(\mathrm{PPh}_{3}\right) \mathrm{AuCl}(2 \mathrm{~mol} \%, 2.9 \mathrm{mg})$ and $\mathrm{AgBF}_{4}$ ( $2 \mathrm{~mol} \%, 1.1 \mathrm{mg}$ ) at $23{ }^{\circ} \mathrm{C}$ for $12 \mathrm{~h}$. The residue was purified by flash chromatography on silica (5\% EtOAc/pentane): $\mathrm{R}_{\mathrm{f}} 0.66$ (20\% EtOAc/hexanes). ${ }^{1} \mathrm{H}$ NMR (360 MHz, CDCl $) \delta 1.32$ (t, $J=$ $7.1 \mathrm{~Hz}, 3 \mathrm{H}$ ), 2.23 (s, $3 \mathrm{H}$ ), 2.51 (s, $3 \mathrm{H}$ ), 4.25 (q, $J=7.1 \mathrm{~Hz}, 2 \mathrm{H}$ ), 6.20 (app. s, $1 \mathrm{H}$ ); ${ }^{13} \mathrm{C}$ NMR

\footnotetext{
${ }^{4}$ Trahanovsky, W. S.; Chou, C.-H.; Cassady, T. J. J. Org. Chem. 1994, 59, 2613-2615.
} 
(90.6 MHz, $\left.\mathrm{CDCl}_{3}\right) \delta$ 13.1, 13.6, 14.4, 59.9, 106.2, 114.0, 149.8, 157.5, 164.3; LRMS (EI) 168 (52\%) $\left[\mathrm{M}^{+}\right], 139$ (100\%) $\left[\mathrm{M}^{+}-\mathrm{CH}_{2} \mathrm{CH}_{3}\right], 123$ (74\%); HRMS 168.0779 [168.0786 calcd for $\left.\mathrm{C}_{9} \mathrm{H}_{12} \mathrm{O}_{3}{ }^{+}\right]$.

Methyl 5-methyl-2-pentylfuran-3-carboxylate (3g). Following the general procedure, 3g (54.0 $\mathrm{mg}, 0.25 \mathrm{mmol}, 77 \%)$ was obtained as a pale yellow oil from $1 \mathbf{g}(70.0 \mathrm{mg}, 0.33 \mathrm{mmol})$. The reaction was conducted in $\mathrm{CH}_{2} \mathrm{Cl}_{2}(1.45 \mathrm{~mL})$ in the presence of $\left(\mathrm{PPh}_{3}\right) \mathrm{AuCl}(2 \mathrm{~mol} \%, 2.9 \mathrm{mg})$ and $\mathrm{AgBF}_{4}(2 \mathrm{~mol} \%, 1.2 \mathrm{mg})$ at $23{ }^{\circ} \mathrm{C}$ for $12 \mathrm{~h}$. The residue was purified by flash chromatography on silica (2\% EtOAc/pentane): $\mathrm{R}_{\mathrm{f}} 0.70$ (20\% EtOAc/hexanes). ${ }^{1} \mathrm{H}$ NMR (360 $\left.\mathrm{MHz}, \mathrm{CDCl}_{3}\right) \delta 0.89$ (t, $\left.J=6.8 \mathrm{~Hz}, 3 \mathrm{H}\right), 1.31-1.34$ (m, $\left.4 \mathrm{H}\right), 1.62-1.66(\mathrm{~m}, 2 \mathrm{H}), 2.24$ (s, $3 \mathrm{H}$ ), 2.91 (t, $J=7.7 \mathrm{~Hz}, 2 \mathrm{H}$ ), 3.79 (s, $3 \mathrm{H}), 6.19$ (s, $1 \mathrm{H}) ;{ }^{13} \mathrm{C} \mathrm{NMR}\left(90.6 \mathrm{MHz}, \mathrm{CDCl}_{3}\right) \delta$ 13.2, 13.9, 22.3, 27.5, 27.8, 31.4, 51.1, 106.1, 113.2, 149.9, 161.9, 164.7; LRMS (EI) 210 (25\%) [M+], 153 (100\%); HRMS 210.1262 [210.1256 calcd for $\mathrm{C}_{12} \mathrm{H}_{18} \mathrm{O}_{3}{ }^{+}$].

Ethyl 2,5-dimethyl-4-phenylfuran-3-carboxylate (3h). Following the general procedure, $3 \mathrm{~h}$ (52.3 mg, $0.22 \mathrm{mmol}, 87 \%)$ was obtained as a colorless oil from $\mathbf{1 h}$ (60.0 $\mathrm{mg}, 0.25 \mathrm{mmol})$. The reaction was conducted in $\mathrm{CH}_{2} \mathrm{Cl}_{2}(1.25 \mathrm{~mL})$ in the presence of $\left(\mathrm{PPh}_{3}\right) \mathrm{AuCl}(2 \mathrm{~mol} \%, 2.5 \mathrm{mg})$ and $\mathrm{AgBF}_{4}(2 \mathrm{~mol} \%, 0.9 \mathrm{mg})$ at $23^{\circ} \mathrm{C}$ for $2 \mathrm{~h}$. The residue was purified by flash chromatography on silica (2\% EtOAc/pentane): $\mathrm{R}_{\mathrm{f}} 0.59$ (20\% EtOAc/hexanes). ${ }^{1} \mathrm{H}$ NMR (360 MHz, $\left.\mathrm{CDCl}_{3}\right) \delta$ 1.10 (t, $J=7.2 \mathrm{~Hz}, 3 \mathrm{H}$ ), 2.21 (s, $3 \mathrm{H}$ ), 2.59 (s, 3 H), 4.13 (q, $J=7.2 \mathrm{~Hz}, 2 \mathrm{H}$ ), 7.25-7.38 (m, 5 $\mathrm{H}) ;{ }^{13} \mathrm{C}$ NMR $\left(90.6 \mathrm{MHz}, \mathrm{CDCl}_{3}\right) \delta 11.7,13.9,14.0,59.7,113.6,121.4,126.7,127.6,130.0$,

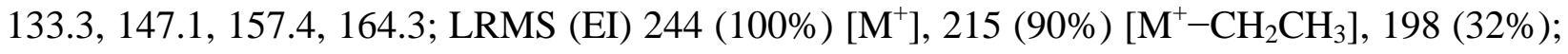
HRMS 244.1095 [244.1099 calcd for $\mathrm{C}_{15} \mathrm{H}_{16} \mathrm{O}_{3}{ }^{+}$].

Ethyl 5-methyl-2,4-diphenylfuran-3-carboxylate (3i). ${ }^{5}$ Following the general procedure, $3 \mathbf{i}$ (63.1 mg, $0.21 \mathrm{mmol}, 90 \%$ ) was obtained as a colorless oil from $1 \mathbf{i}$ (70.1 $\mathrm{mg}, 0.23 \mathrm{mmol}$ ). The reaction was conducted in $\mathrm{CH}_{2} \mathrm{Cl}_{2}(1.15 \mathrm{~mL})$ in the presence of $\left(\mathrm{PPh}_{3}\right) \mathrm{AuCl}(2 \mathrm{~mol} \%, 2.3 \mathrm{mg})$ and $\mathrm{AgBF}_{4}(2 \mathrm{~mol} \%, 0.9 \mathrm{mg})$ at $23{ }^{\circ} \mathrm{C}$ for $4 \mathrm{~h}$. The residue was purified by flash chromatography on silica (10\% EtOAc/pentane): $\mathrm{R}_{\mathrm{f}} 0.57$ (20\% EtOAc/hexanes). ${ }^{1} \mathrm{H}$ NMR (360 MHz, $\left.\mathrm{CDCl}_{3}\right) \delta$ 0.98 (t, $J=7.0 \mathrm{~Hz}, 3$ H), 2.33 (s, 3 H), 4.09 (q, $J=7.0$ Hz, 2 H), 7.31-7.42 (m, 8 H), 7.82-7.83

\footnotetext{
${ }^{5}$ Ficini, J.; Claeys, M.; Depezay, J. C. Tetrahedron Lett. 1973, 14, 3357-3359.
} 
(m, $2 \mathrm{H}) ;{ }^{13} \mathrm{C}$ NMR $\left(90.6 \mathrm{MHz}, \mathrm{CDCl}_{3}\right) \delta 12.0,13.6,60.5,114.9,122.9,126.9,127.6,127.9$, 128.2, 128.7, 129.6, 130.1, 133.0, 148.4, 153.9, 164.6; LRMS (EI) 306 (100\%) [M+'], 261 (28\%), 105(42\%); HRMS 306.1253 [306.1256 calcd for $\mathrm{C}_{20} \mathrm{H}_{18} \mathrm{O}_{3}{ }^{+}$].

Ethyl 5-methyl-4-phenylfuran-3-carboxylate (3j). Following the general procedure, 3j (58.8 $\mathrm{mg}, 0.25 \mathrm{mmol}$, 84\%) was obtained as a colorless oil from $\mathbf{1 j}$ (70.0 $\mathrm{mg}, 0.30 \mathrm{mmol})$. The reaction was conducted in benzene $(1.50 \mathrm{~mL})$ in the presence of $\left(\mathrm{PPh}_{3}\right) \mathrm{AuCl}(2 \mathrm{~mol} \%, 3.0 \mathrm{mg})$ and $\mathrm{AgBF}_{4}$ ( $2 \mathrm{~mol} \%, 1.2 \mathrm{mg}$ ) at $23{ }^{\circ} \mathrm{C}$ for $4 \mathrm{~h}$. The residue was purified by flash chromatography on silica (5\% EtOAc/pentane): $\mathrm{R}_{\mathrm{f}} 0.62$ (20\% EtOAc/hexanes). ${ }^{1} \mathrm{H}$ NMR (360 MHz, $\left.\mathrm{CDCl}_{3}\right) \delta 0.98$ (t, $J=$ $7.0 \mathrm{~Hz}, 3 \mathrm{H}$ ), 2.33 (s, 3 H), 4.09 (q, J = 7.0 Hz, 2 H), 7.31-7.42 (m, 8 H), 7.82-7.83 (m, $2 \mathrm{H})$; ${ }^{13} \mathrm{C}$ NMR (90.6 MHz, $\left.\mathrm{CDCl}_{3}\right) \delta 12.0,13.6,60.5,114.9,122.9,126.9,127.6,127.9,128.2,128.7$, 129.6, 130.1, 133.0, 148.4, 153.9, 164.6; LRMS (EI) 230 (100\%) [M+1, 185 (67\%), 105 (45\%), 84 (56\%); HRMS 230.0939 [230.0943 calcd for $\mathrm{C}_{14} \mathrm{H}_{14} \mathrm{O}_{3}{ }^{+}$].

Ethyl 4-(2-methoxyphenyl)-5-methyl-2-phenylfuran-3-carboxylate (3k). Following the general procedure, 3k (69.1 mg, $0.25 \mathrm{mmol}$, 99\%) was obtained as a colorless oil from 1k (70.0 $\mathrm{mg}, 0.26 \mathrm{mmol})$. The reaction was conducted in $\mathrm{CH}_{2} \mathrm{Cl}_{2}(1.30 \mathrm{~mL})$ in the presence of $\left(\mathrm{PPh}_{3}\right) \mathrm{AuCl}$ (2 mol\%, $2.3 \mathrm{mg}$ ) and $\mathrm{AgBF}_{4}\left(2 \mathrm{~mol} \%, 1.0 \mathrm{mg}\right.$ ) at $23^{\circ} \mathrm{C}$ for $3 \mathrm{~h}$. The residue was purified by flash chromatography on silica (5\% EtOAc/pentane): $\mathrm{R}_{\mathrm{f}} 0.39$ (20\% EtOAc/hexanes). ${ }^{1} \mathrm{H}$ NMR (360 MHz, $\mathrm{CDCl}_{3}$ ): $\delta 1.03$ (t, $J=7.2 \mathrm{~Hz}, 3 \mathrm{H}$ ), 2.17 (s, $3 \mathrm{H}$ ), 2.55 (s, $3 \mathrm{H}$ ), 3.77 (s, $3 \mathrm{H}$ ), 4.06 (q, $J=7.2 \mathrm{~Hz}, 2 \mathrm{H}$ ), 6.89 (d, $J=8.2,0.8 \mathrm{~Hz}, 1 \mathrm{H}$ ), 6.96 (dt, $J=0.8,7.5 \mathrm{~Hz}, 1 \mathrm{H}$ ), 7.13 (dd, $J=7.5$, $1.8 \mathrm{~Hz}, 1 \mathrm{H}), 7.28$ (dt, $J=8.2,1.8 \mathrm{~Hz}, 1 \mathrm{H}) ;{ }^{13} \mathrm{C} \mathrm{NMR}\left(90.6 \mathrm{MHz}, \mathrm{CDCl}_{3}\right): \delta 11.8,13.8,13.9$, 55.3, 59.5, 110.3, 114.5, 117.3, 120.1, 122.5, 128.5, 131.1, 147.1, 156.6, 157.4, 164.5; LRMS (EI) 274 (100\%) [M+], 213 (92\%), 43 (68\%); HRMS 274.1206 [274.1205 calcd for $\mathrm{C}_{16} \mathrm{H}_{18} \mathrm{O}_{4}{ }^{+}$].

Ethyl 5-methyl-2-phenyl-4-(thiophen-2-yl)furan-3-carboxylate (3l). Following the general procedure, $3 \mathbf{l}$ (53.2 mg, $0.17 \mathrm{mmol}$, 89\%) was obtained as a colorless oil from 11 (60.0 mg, 0.19 mmol). The reaction was conducted in $\mathrm{CH}_{2} \mathrm{Cl}_{2}(0.95 \mathrm{~mL})$ in the presence of $\left(\mathrm{PPh}_{3}\right) \mathrm{AuCl}(2$ mol\%, $2.0 \mathrm{mg}$ ) and $\mathrm{AgBF}_{4}\left(2 \mathrm{~mol} \%, 1.0 \mathrm{mg}\right.$ ) at $23^{\circ} \mathrm{C}$ for $4 \mathrm{~h}$. The residue was purified by flash chromatography on silica (10\% EtOAc/pentane): $\mathrm{R}_{\mathrm{f}} 0.58$ (20\% EtOAc/hexanes). ${ }^{1} \mathrm{H}$ NMR (360 MHz, $\mathrm{CDCl}_{3}$ ): $\delta 1.08$ (t, $J=7.3 \mathrm{~Hz}, 3 \mathrm{H}$ ), 2.36 (s, $3 \mathrm{H}$ ), 4.15 (q, $J=7.3 \mathrm{~Hz}, 2 \mathrm{H}$ ), 7.11 (dd, $J=$ $5.0 \mathrm{~Hz}, J=1.2 \mathrm{~Hz}, 1 \mathrm{H}), 7.21-7.24(\mathrm{~m}, 1 \mathrm{H}), 7.33-7.42$ (m, $4 \mathrm{H}), 7.78-7.81$ (m, $2 \mathrm{H}) ;{ }^{13} \mathrm{C}$ NMR 
(90.6 MHz, $\left.\mathrm{CDCl}_{3}\right): \delta 12.2,13.7,60.6,114.8,117.9,123.1,124.6,127.6,128.1,128.7,129.2$, 130.1, 132.6, 148.8, 153.9, 164.6; LRMS (EI) 312 (100\%) [ $\left.\mathrm{M}^{+}\right], 284$ (12\%) $\left[\mathrm{M}^{+}-\mathrm{C}_{2} \mathrm{H}_{4}\right], 267$ (20\%); HRMS 312.0826 [312.0820 calcd for $\mathrm{C}_{18} \mathrm{H}_{16} \mathrm{O}_{3} \mathrm{~S}^{+}$].

Ethyl 5-methyl-2-phenyl-4-(pyridin-2-yl)furan-3-carboxylate (3m). Following the general procedure, 3m (49.0 mg, $0.16 \mathrm{mmol}$, 82\%) was obtained as a pale yellow oil from $\mathbf{1 m}$ (60.0 mg, $0.19 \mathrm{mmol})$. The reaction was conducted in benzene $(2.0 \mathrm{~mL})$ in the presence of $\left(\mathrm{PPh}_{3}\right) \mathrm{AuCl}(2$ mol\%, $2.0 \mathrm{mg}$ ) and $\mathrm{AgBF}_{4}(2 \mathrm{~mol} \%, 0.9 \mathrm{mg})$ at $23{ }^{\circ} \mathrm{C}$ for $48 \mathrm{~h}$. The residue was purified by flash chromatography on silica (20\% EtOAc/pentane): $\mathrm{R}_{\mathrm{f}} 0.15$ (20\% EtOAc/hexanes). ${ }^{1} \mathrm{H}$ NMR (360 MHz, $\mathrm{CDCl}_{3}$ ): $\delta 1.02$ (t, $J=7.3 \mathrm{~Hz}, 3 \mathrm{H}$ ), 2.44 (s, $3 \mathrm{H}$ ), 4.12 (q, $J=7.3 \mathrm{~Hz}, 2 \mathrm{H}$ ), 7.21 (ddd, $J=$ $7.5 \mathrm{~Hz}, J=5.0 \mathrm{~Hz}, J=1.1 \mathrm{~Hz}, 1 \mathrm{H}), 7.34-7.46$ (m, $4 \mathrm{H}), 7.71(\mathrm{dt}, J=1.8 \mathrm{~Hz}, J=7.7 \mathrm{~Hz}, 1 \mathrm{H})$, 7.83-7.87 (m, $2 \mathrm{H}), 8.63-8.67$ (m, $1 \mathrm{H}) ;{ }^{13} \mathrm{C}$ NMR (90.6 MHz, $\left.\mathrm{CDCl}_{3}\right): \delta 12.4,13.7,60.5,114.6$, 121.6, 122.8, 124.1, 127.6, 128.2, 128.8, 129.9, 135.8, 149.3, 150.1, 152.6, 153.9, 164.5; LRMS (EI) 307 (25\%) $\left[\mathrm{M}^{+}\right], 234$ (44\%), 105 (100\%); HRMS 307.1211 [307.1208 calcd for $\left.\mathrm{C}_{19} \mathrm{H}_{17} \mathrm{NO}_{3}^{+}\right]$.

Ethyl 4-(cyclohexylmethyl)-5-methyl-2-phenylfuran-3-carboxylate (3n). Following the general procedure, 3n (51.0 mg, $0.15 \mathrm{mmol}$, 73\%) was obtained as a colorless oil from 1n (70.0 $\mathrm{mg}, 0.21 \mathrm{mmol})$. The reaction was conducted in $\mathrm{CH}_{2} \mathrm{Cl}_{2}(1.0 \mathrm{~mL})$ in the presence of $\left(\mathrm{PPh}_{3}\right) \mathrm{AuCl}$ (2 mol\%, $1.9 \mathrm{mg}$ ) and $\mathrm{AgBF}_{4}\left(2 \mathrm{~mol} \%, 1.0 \mathrm{mg}\right.$ ) at $23{ }^{\circ} \mathrm{C}$ for $5 \mathrm{~h}$. The residue was purified by flash chromatography on silica (2\% EtOAc/pentane): $\mathrm{R}_{\mathrm{f}} 0.69$ (20\% EtOAc/hexanes). ${ }^{1} \mathrm{H}$ NMR (360 MHz, $\left.\mathrm{CDCl}_{3}\right): \delta$ 0.72-1.02 (m, $\left.2 \mathrm{H}\right), 1.06-1.19(\mathrm{~m}, 3 \mathrm{H}), 1.28(\mathrm{t}, J=7.0 \mathrm{~Hz}, 3 \mathrm{H})$, 1.42-1.50 (m, 1 H), 1.61-1.72 (m, 5 H), 2.27 (s, 3 H), 2.42 (d, $J=7.0$ Hz, 2 H), 4.27 (q, $J=7.0$ $\mathrm{Hz}, 2 \mathrm{H}), 7.36-7.38$ (m, $3 \mathrm{H}), 7.73$ (d, $J=6.8 \mathrm{~Hz}, 2 \mathrm{H}) ;{ }^{13} \mathrm{C}$ NMR (90.6 MHz, $\left.\mathrm{CDCl}_{3}\right): \delta$ 11.7, 14.0, 26.4, 26.6, 32.0, 33.3, 39.0, 60.2, 114.4, 119.6, 127.9, 128.1, 128.5, 130.7, 148.4, 154.9, 164.9; LRMS (EI) 326 (75\%) [M+], 244 (100\%), 43 (92\%); HRMS 326.1878 [326.1882 calcd for $\left.\mathrm{C}_{21} \mathrm{H}_{26} \mathrm{O}_{3}^{+}\right]$.

\section{Ethyl 4-(2-tert-butyldimethylsilyloxyethyl)-5-methyl-2-phenylfuran-3-carboxylate (30).}

Following the general procedure, $3 \mathbf{0}(43.2 \mathrm{mg}, 0.11 \mathrm{mmol}, 72 \%)$ was obtained as a colorless oil from 10 (60.0 mg, $0.15 \mathrm{mmol})$. The reaction was conducted in $\mathrm{CH}_{2} \mathrm{Cl}_{2}(0.75 \mathrm{~mL})$ in the presence of $\left(\mathrm{PPh}_{3}\right) \mathrm{AuCl}(2 \mathrm{~mol} \%, 1.7 \mathrm{mg})$ and $\mathrm{AgBF}_{4}(2 \mathrm{~mol} \%, 0.9 \mathrm{mg})$ at $23^{\circ} \mathrm{C}$ for $3 \mathrm{~h}$. The residue was 
purified by flash chromatography on silica (5\% Et $2 \mathrm{O} /$ pentane): $\mathrm{R}_{\mathrm{f}} 0.71$ (20\% EtOAc/hexanes). ${ }^{1} \mathrm{H}$ NMR (360 MHz, $\left.\mathrm{CDCl}_{3}\right): \delta 0.00$ (s, $\left.6 \mathrm{H}\right), 0.87$ (s, $\left.9 \mathrm{H}\right), 1.26$ (t, $\left.J=7.0 \mathrm{~Hz}, 3 \mathrm{H}\right), 2.30$ (s, $3 \mathrm{H}$ ), 2.80 (t, $J=6.7 \mathrm{~Hz}, 2 \mathrm{H}), 3.76$ (t, $J=6.7 \mathrm{~Hz}, 2 \mathrm{H}), 4.26$ (q, $J=7.0 \mathrm{~Hz}, 2 \mathrm{H}), 7.36-7.39$ (m, $3 \mathrm{H})$, 7.70-7.73 (m, $2 \mathrm{H})$; ${ }^{13} \mathrm{C}$ NMR (90.6 MHz, $\mathrm{CDCl}_{3}$ ): $\delta$-5.4, 11.6, 14.0, 18.4, 25.9, 28.1, 60.2, 63.3, 114.2, 117.6, 127.9, 128.3, 128.6, 130.7, 149.4, 155.2, 164.5; LRMS (EI) 388 (1\%) [M ${ }^{+}$, 331 (7\%) $\left[\mathrm{M}^{+}-\mathrm{C}_{4} \mathrm{H}_{9}\right.$ ], 219 (24\%), 147 (41\%), 84 (100\%); HRMS 331.1363 [331.1365 calcd for fragment $\mathrm{C}_{18} \mathrm{H}_{23} \mathrm{O}_{4} \mathrm{Si}^{+}$]; HRMS 388.2077 [388.2070 calcd for $\mathrm{C}_{22} \mathrm{H}_{32} \mathrm{O}_{4} \mathrm{Si}^{+}$].

Ethyl 4-(tert-butyldimethylsilyl)-2,5-dimethylfuran-3-carboxylate (3p). Following the general procedure, 3p (57.8 mg, $0.20 \mathrm{mmol}$, 83\%) was obtained as a colorless oil from 1p (70.0 mg, 0.25 $\mathrm{mmol})$. The reaction was conducted in $\mathrm{CH}_{2} \mathrm{Cl}_{2}(1.25 \mathrm{~mL})$ in the presence of $\left(\mathrm{PPh}_{3}\right) \mathrm{AuCl}(2$ mol\%, $2.5 \mathrm{mg}$ ) and $\mathrm{AgBF}_{4}$ (2 mol\%, $1.0 \mathrm{mg}$ ) at $23{ }^{\circ} \mathrm{C}$ for $4 \mathrm{~h}$. The residue was purified by flash chromatography on silica (2\% EtOAc/pentane): $\mathrm{R}_{\mathrm{f}} 0.80$ (20\% EtOAc/hexanes). ${ }^{1} \mathrm{H}$ NMR (360 MHz, $\mathrm{CDCl}_{3}$ ): $\delta 0.26$ (s, $\left.6 \mathrm{H}\right), 0.91$ (s, $\left.9 \mathrm{H}\right), 1.33$ (t, J = $\left.7.3 \mathrm{~Hz}, 3 \mathrm{H}\right), 2.29$ (s, $\left.3 \mathrm{H}\right), 2.46$ (s, $3 \mathrm{H}$ ), 4.25 (q, $J=7.3 \mathrm{~Hz}, 2 \mathrm{H}$ ); ${ }^{13} \mathrm{C}$ NMR (90.6 MHz, $\mathrm{CDCl}_{3}$ ): $\delta-2.6,13.9,14.4,15.2,18.5,27.1$,

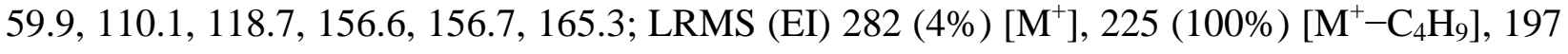
(95\%), 75 (68\%); HRMS 282.1653 [282.1651 calcd for $\mathrm{C}_{15} \mathrm{H}_{26} \mathrm{O}_{3} \mathrm{Si}^{+}$].

Ethyl 5-ethyl-4-methyl-2-phenylfuran-3-carboxylate (3q). ${ }^{6}$ Following the general procedure, 3q (27.3 mg, $0.10 \mathrm{mmol}$, 45\%) was obtained as a pale yellow oil from 1q (60.0 mg, $0.23 \mathrm{mmol})$. The reaction was conducted in $\mathrm{CH}_{2} \mathrm{Cl}_{2}(1.20 \mathrm{~mL})$ in the presence of $\left(\mathrm{PPh}_{3}\right) \mathrm{AuCl}(2 \mathrm{~mol} \%, 2.4$ $\mathrm{mg})$ and $\mathrm{AgBF}_{4}(2 \mathrm{~mol} \%, 1.0 \mathrm{mg})$ at $38{ }^{\circ} \mathrm{C}$ for $24 \mathrm{~h}$. The residue was purified by flash chromatography on silica (2\% EtOAc/pentane): $\mathrm{R}_{\mathrm{f}} 0.67$ (20\% EtOAc/hexanes). ${ }^{1} \mathrm{H}$ NMR (360 $\left.\mathrm{MHz}, \mathrm{CDCl}_{3}\right): \delta 1.24(\mathrm{t}, J=7.7 \mathrm{~Hz}, 3 \mathrm{H}), 1.29(\mathrm{t}, J=7.0 \mathrm{~Hz}, 3 \mathrm{H}), 2.13(\mathrm{~s}, 3 \mathrm{H}), 2.26$ (q, $J=7.7$ Hz, 2 H), 4.27 (q, J = 7.0 Hz, 2 H), 7.38-7.39 (m, 3 H), 7.74-7.77 (m, $2 \mathrm{H})$; ${ }^{13} \mathrm{C}$ NMR (90.6 $\mathrm{MHz}_{\mathrm{CDCl}}$ ): $\delta$ 9.8, 12.8, 14.1, 19.2, 60.2, 114.7, 115.4, 127.9, 128.2, 128.6, 130.7, 152.7, 154.9, 164.8; LRMS (EI) 258 (5\%) [M+], 220 (12\%), 185 (25\%), 147 (28\%), 105 (100\%); HRMS 258.1249 [258.1256 calcd for $\mathrm{C}_{16} \mathrm{H}_{18} \mathrm{O}_{3}{ }^{+}$].

\footnotetext{
${ }^{6}$ Antonioletti, R.; Cecchini, C.; Ciani, B.; Magnanti, S. Tetrahedron Lett. 1995, 36, 9019-9022.
} 
¿্ণ
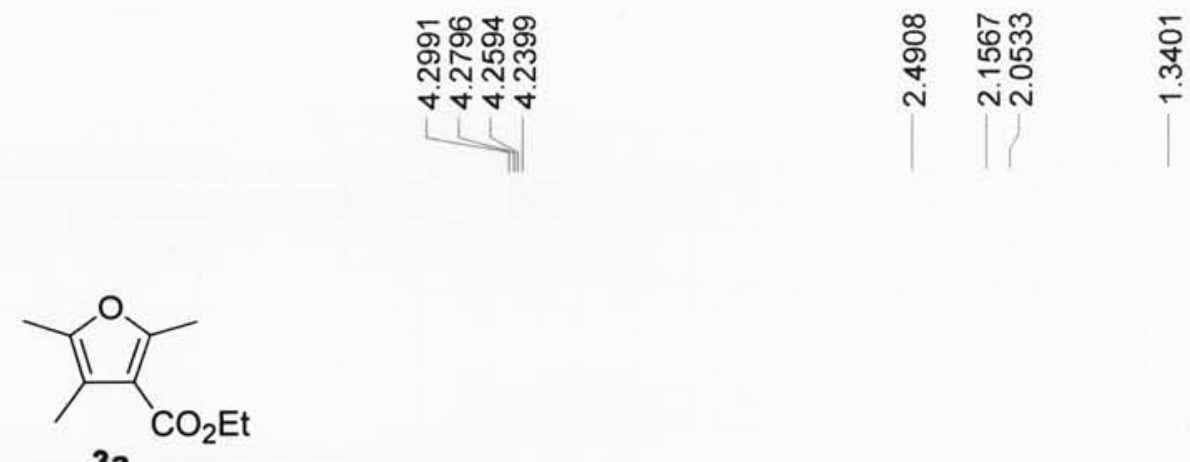

$3 a$
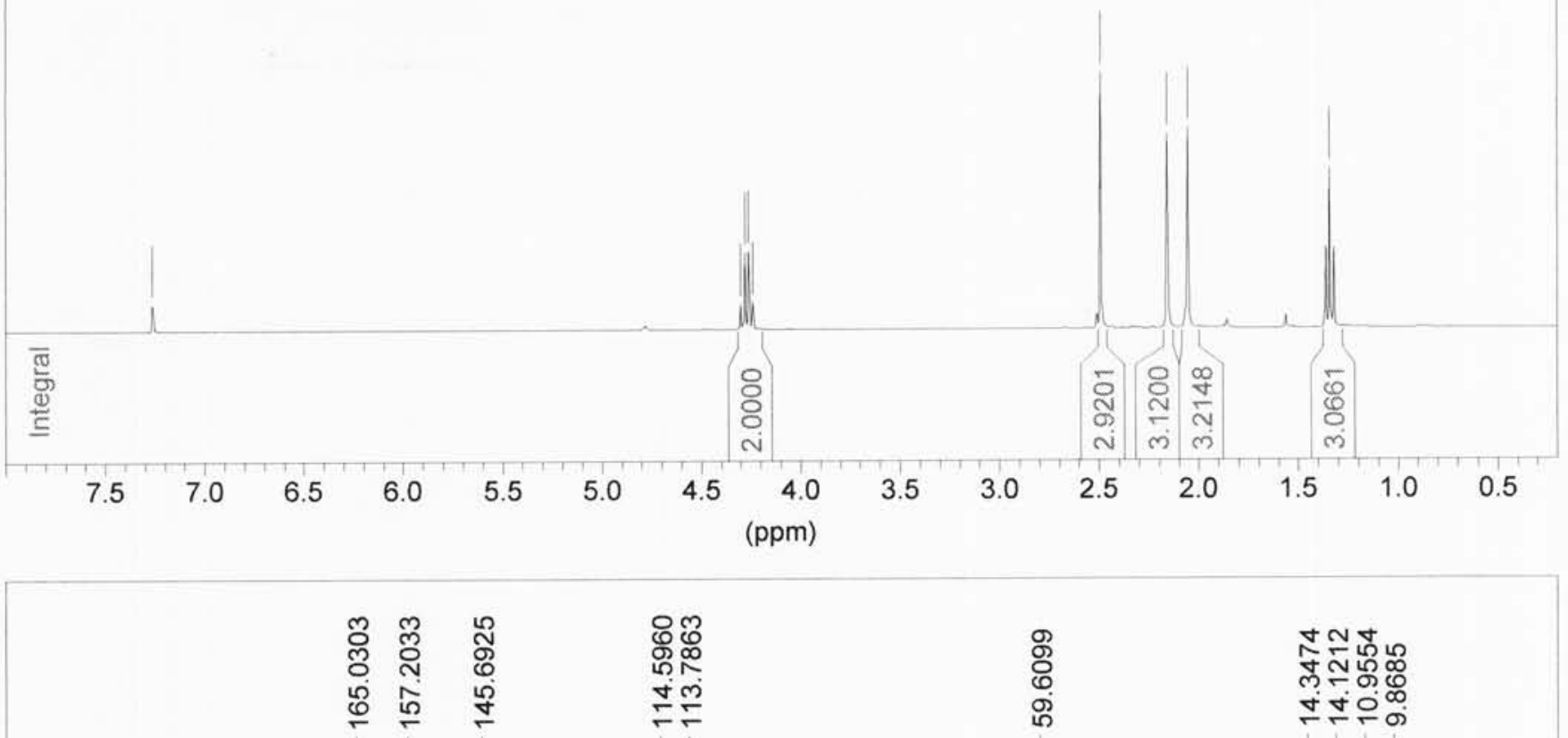

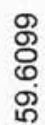

N뉴뉴

लำน

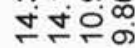

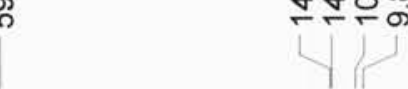

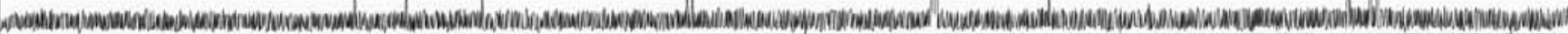



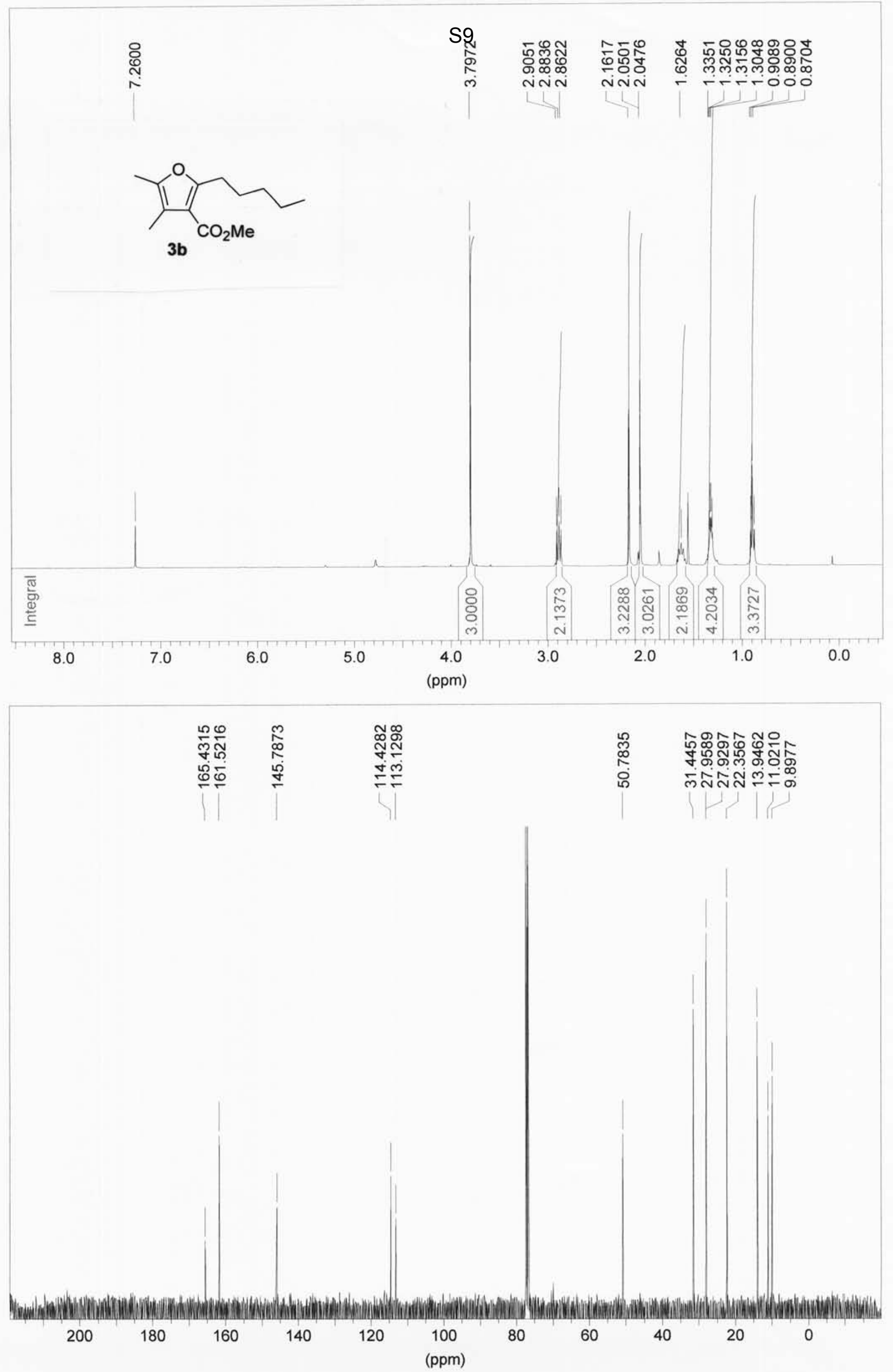

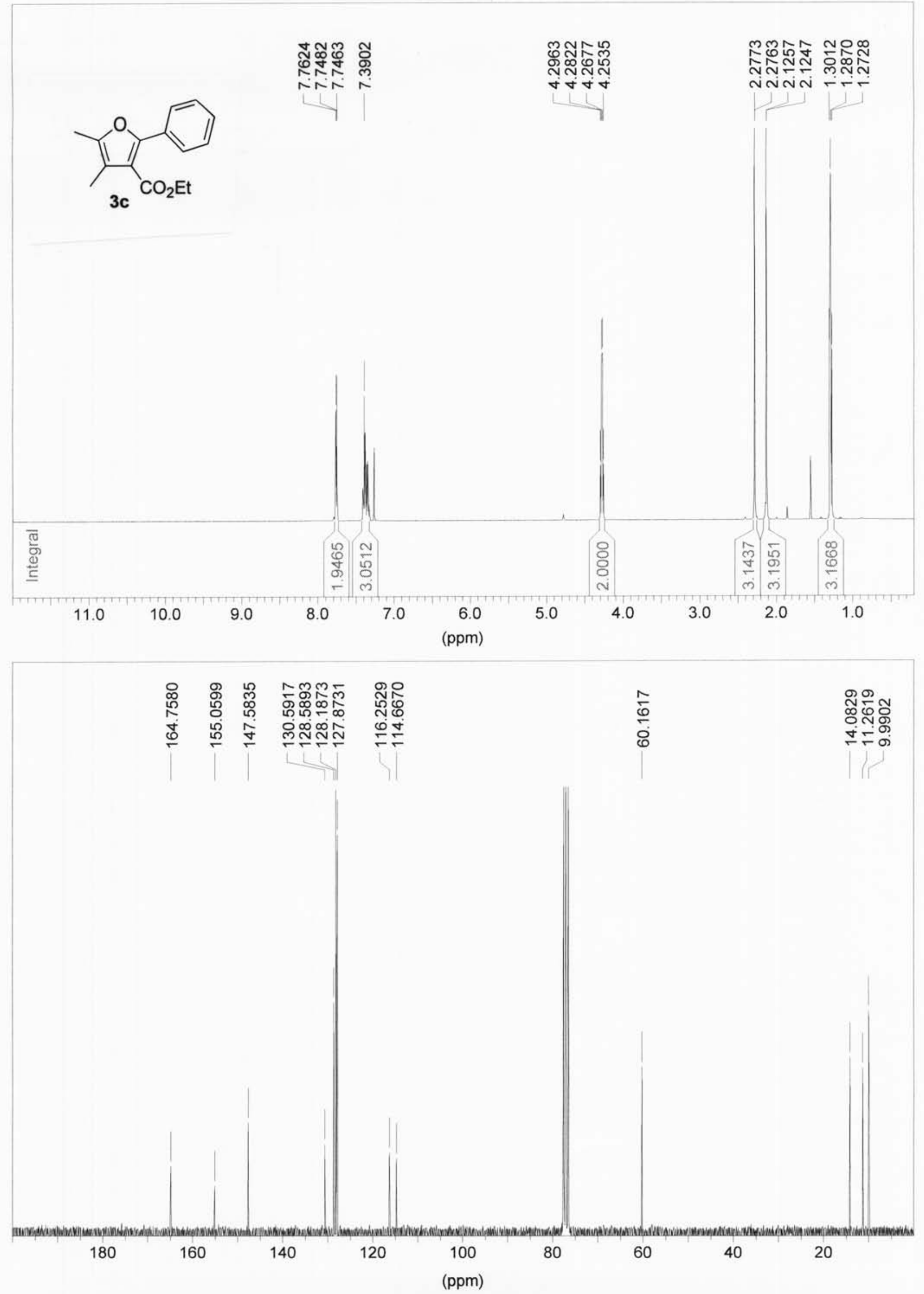

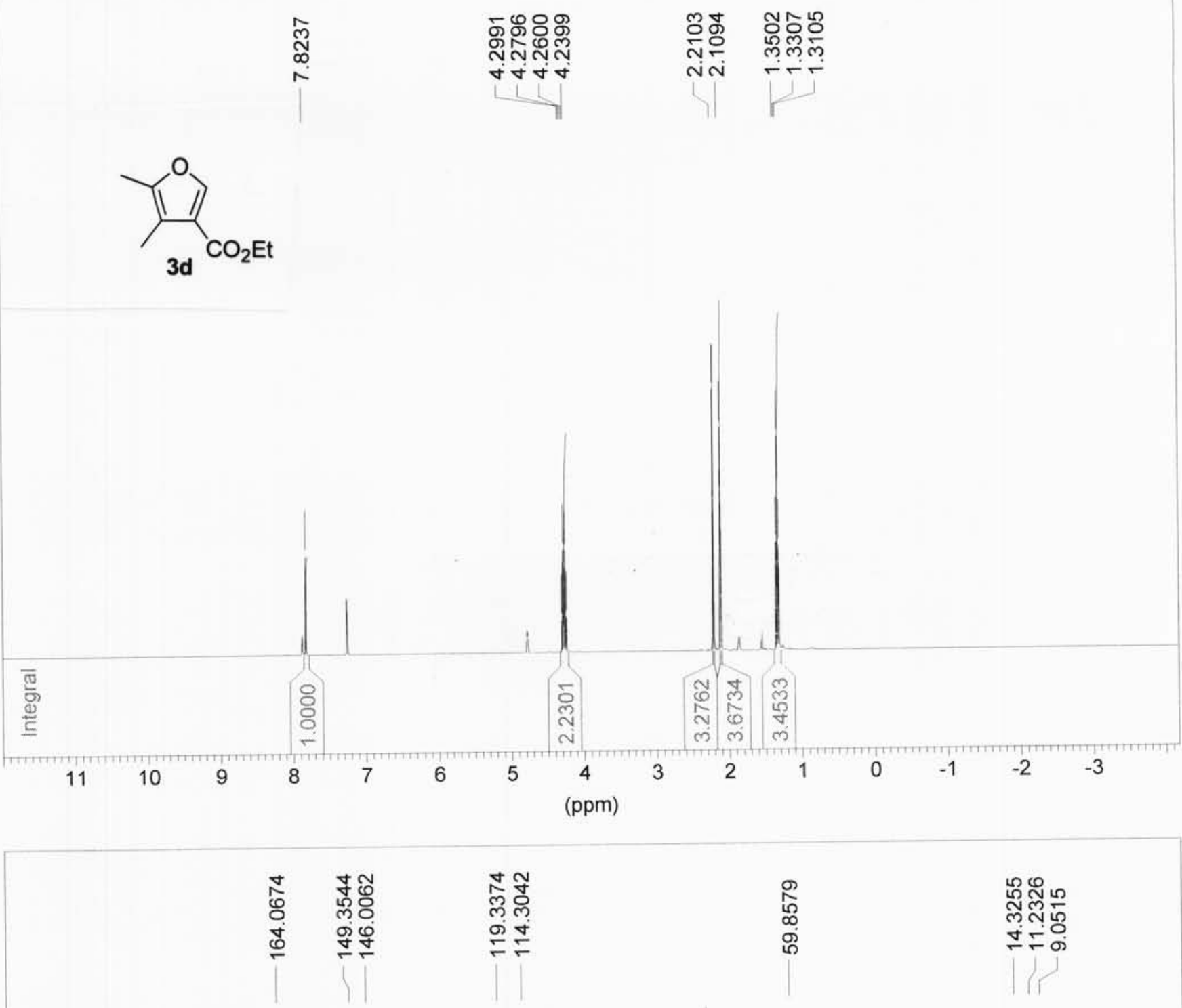

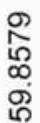

ก๊ำ

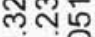

ค) $\quad \forall=0$

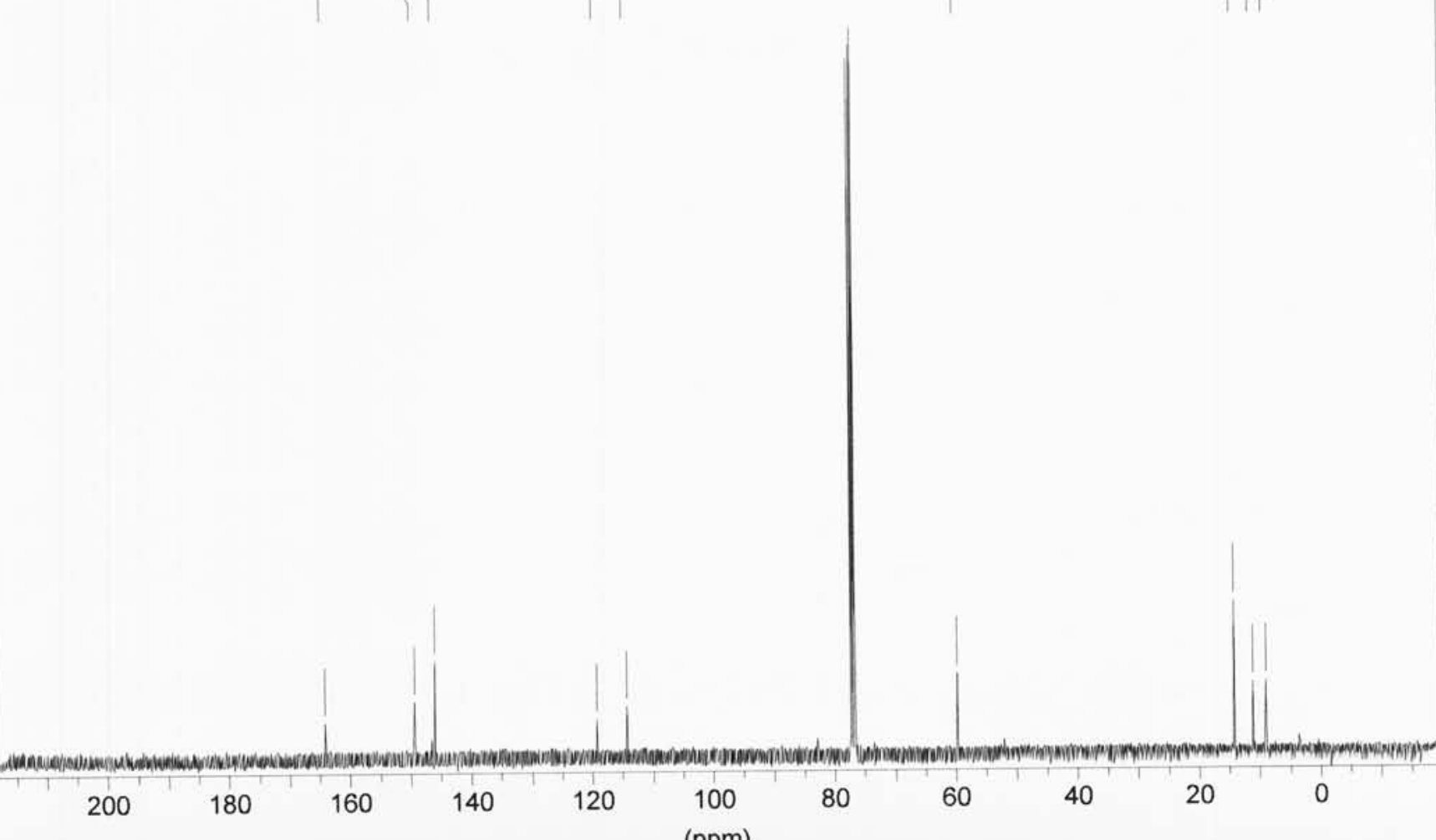

(ppm) 

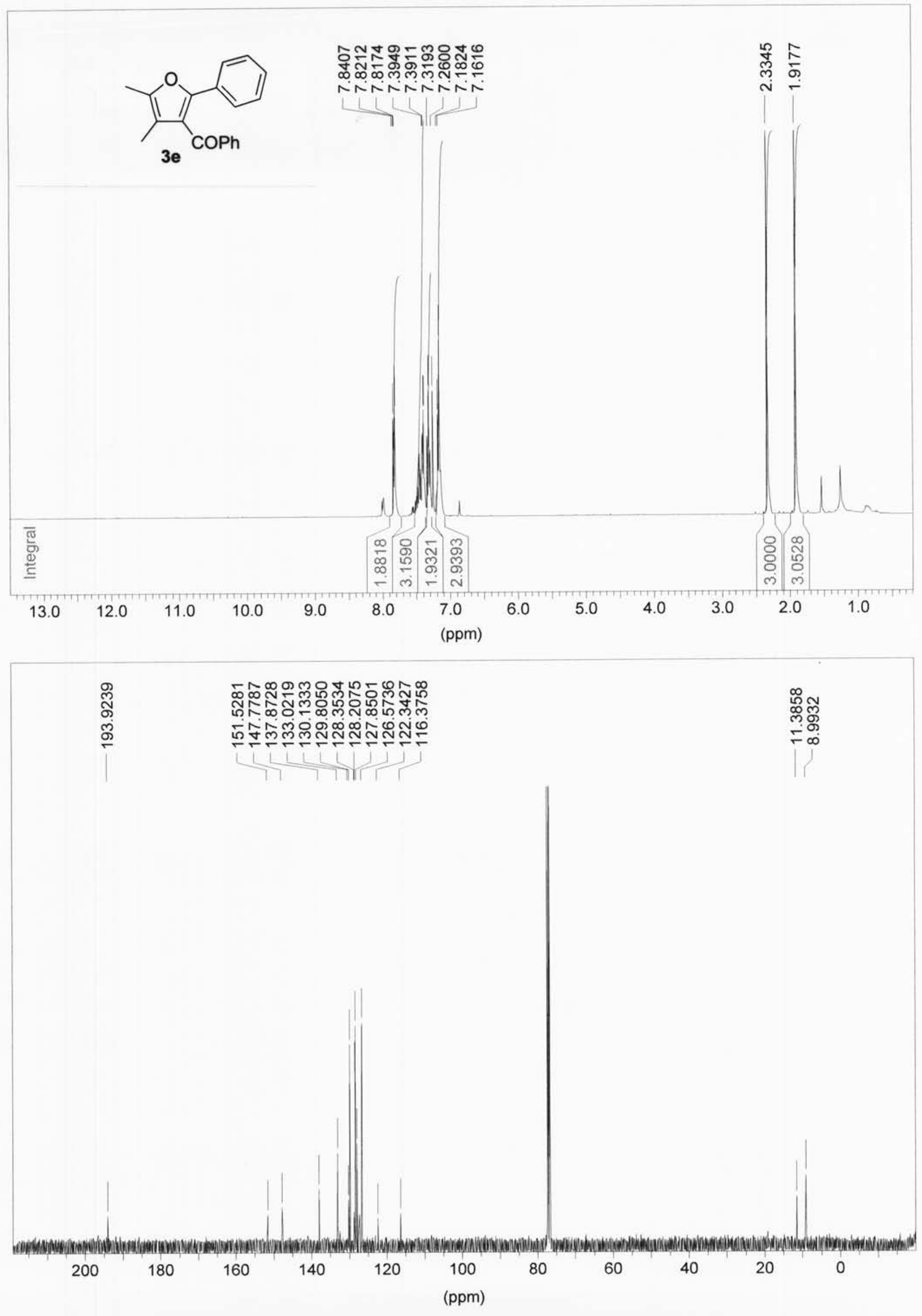

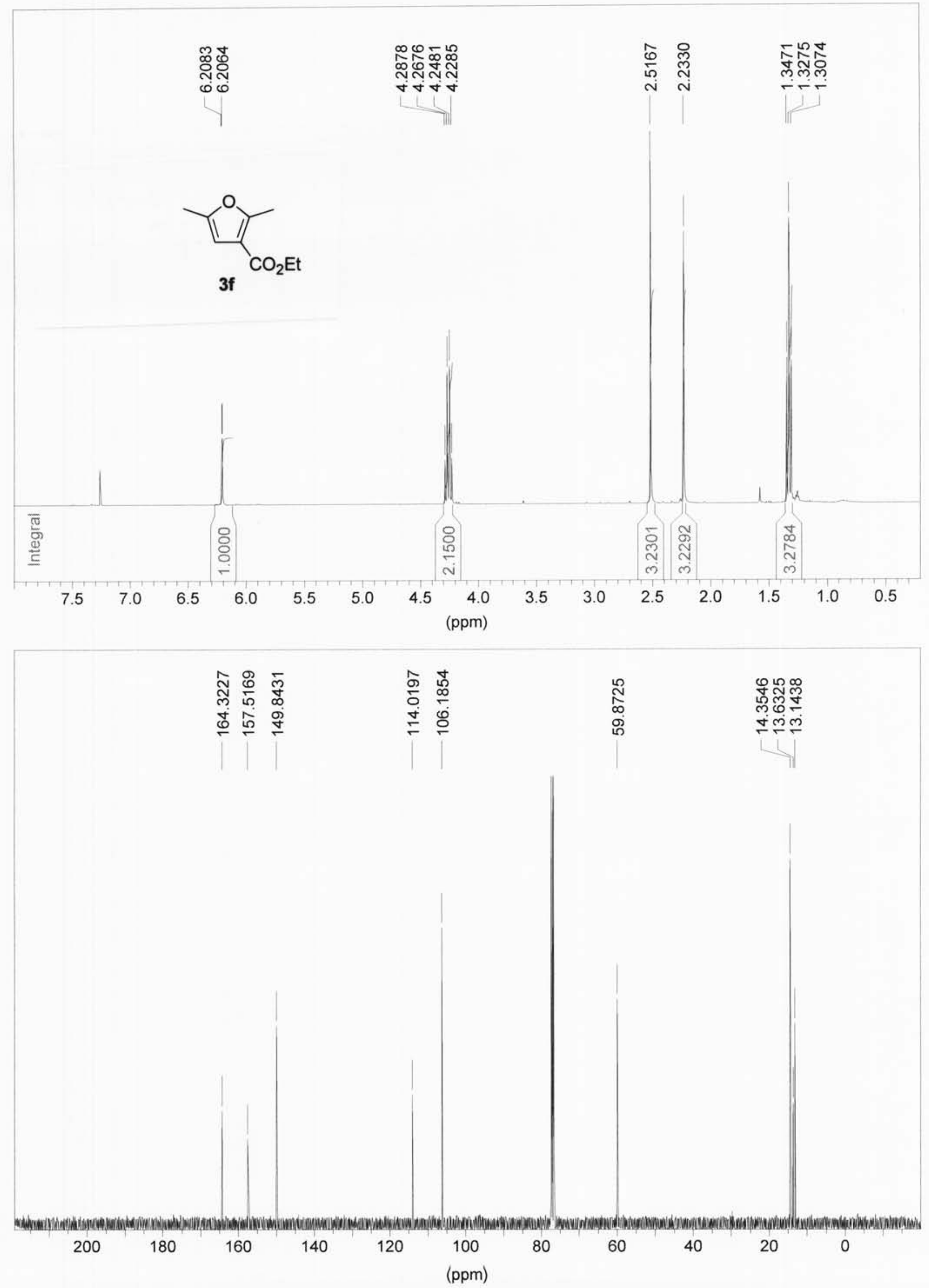

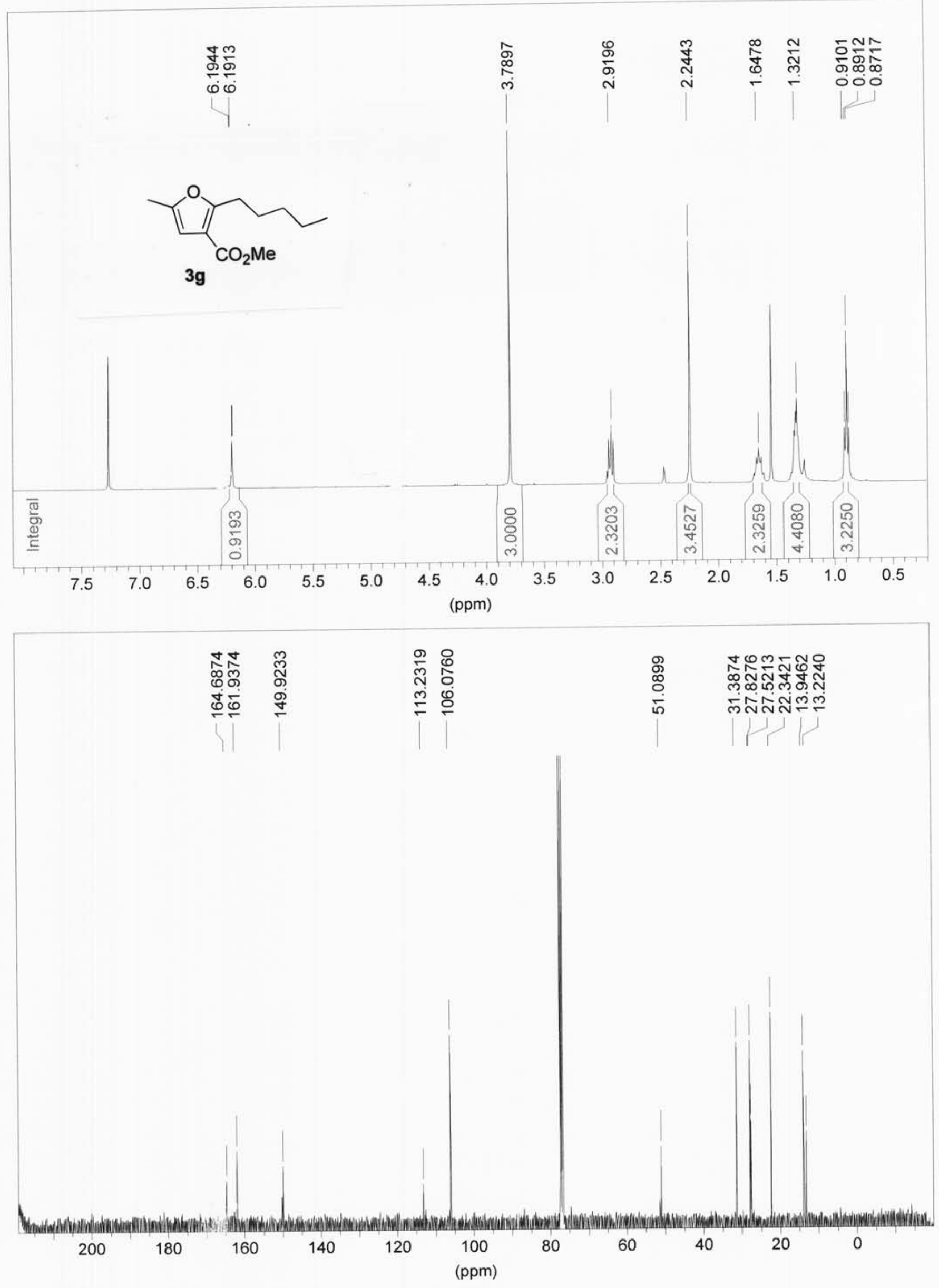

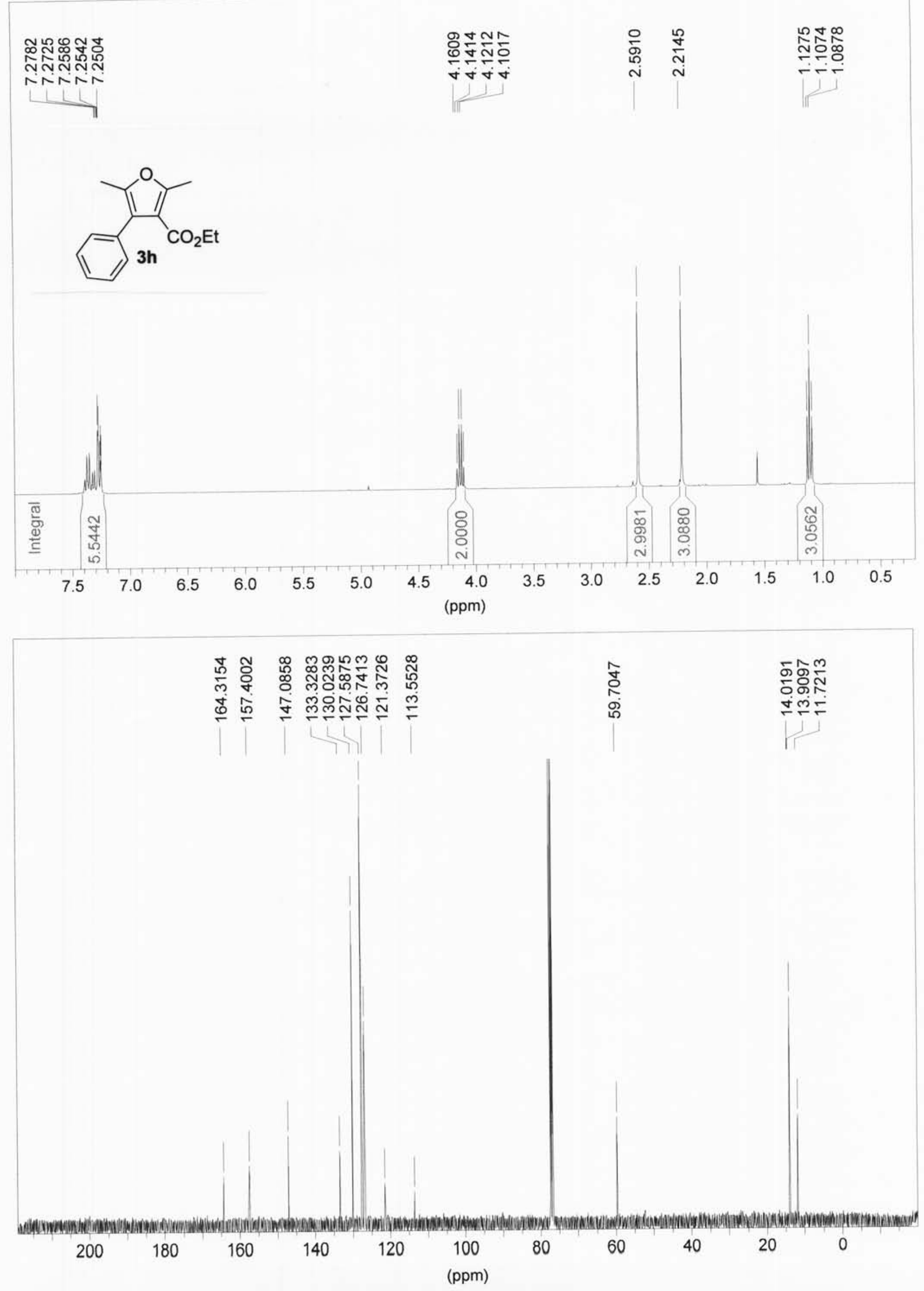

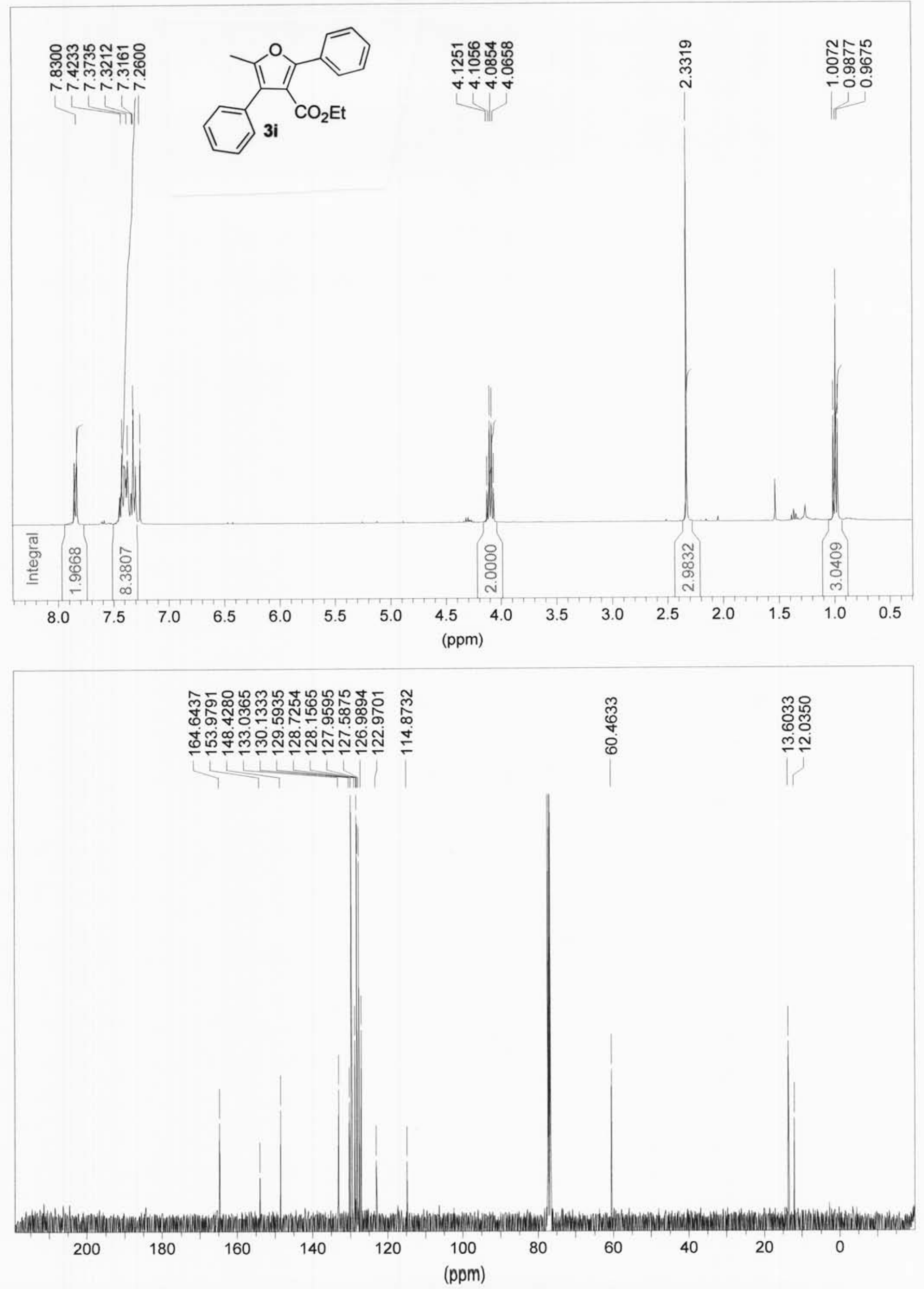


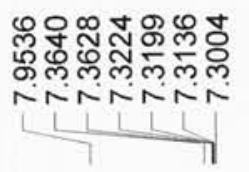

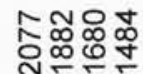

幽元
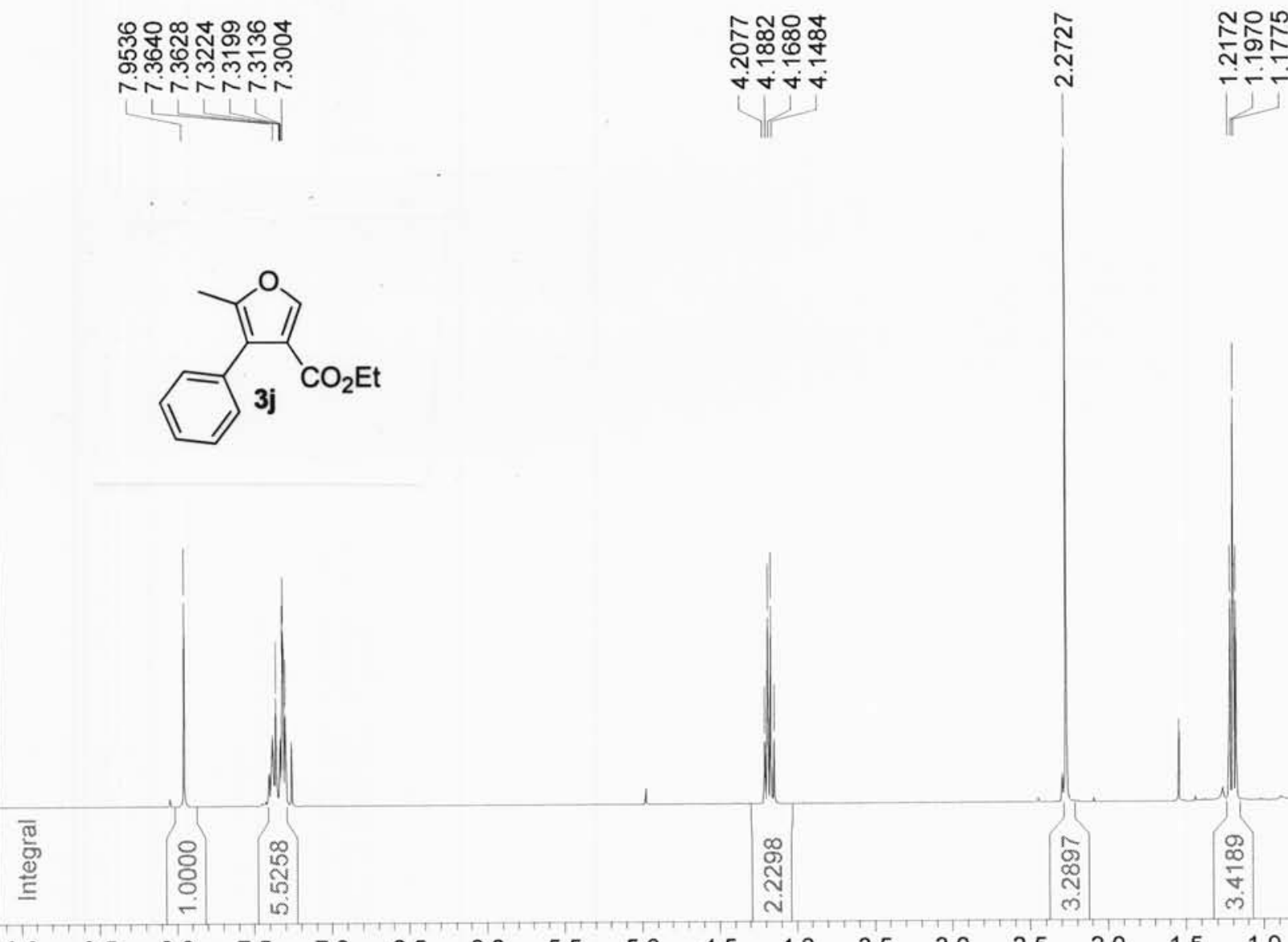

$\begin{array}{llllllllll}9.0 & 8.5 & 8.0 & 7.5 & 7.0 & 6.5 & 6.0 & 5.5 & 5.0 & \begin{array}{l}4.5 \\ (\mathrm{ppm})\end{array}\end{array}$

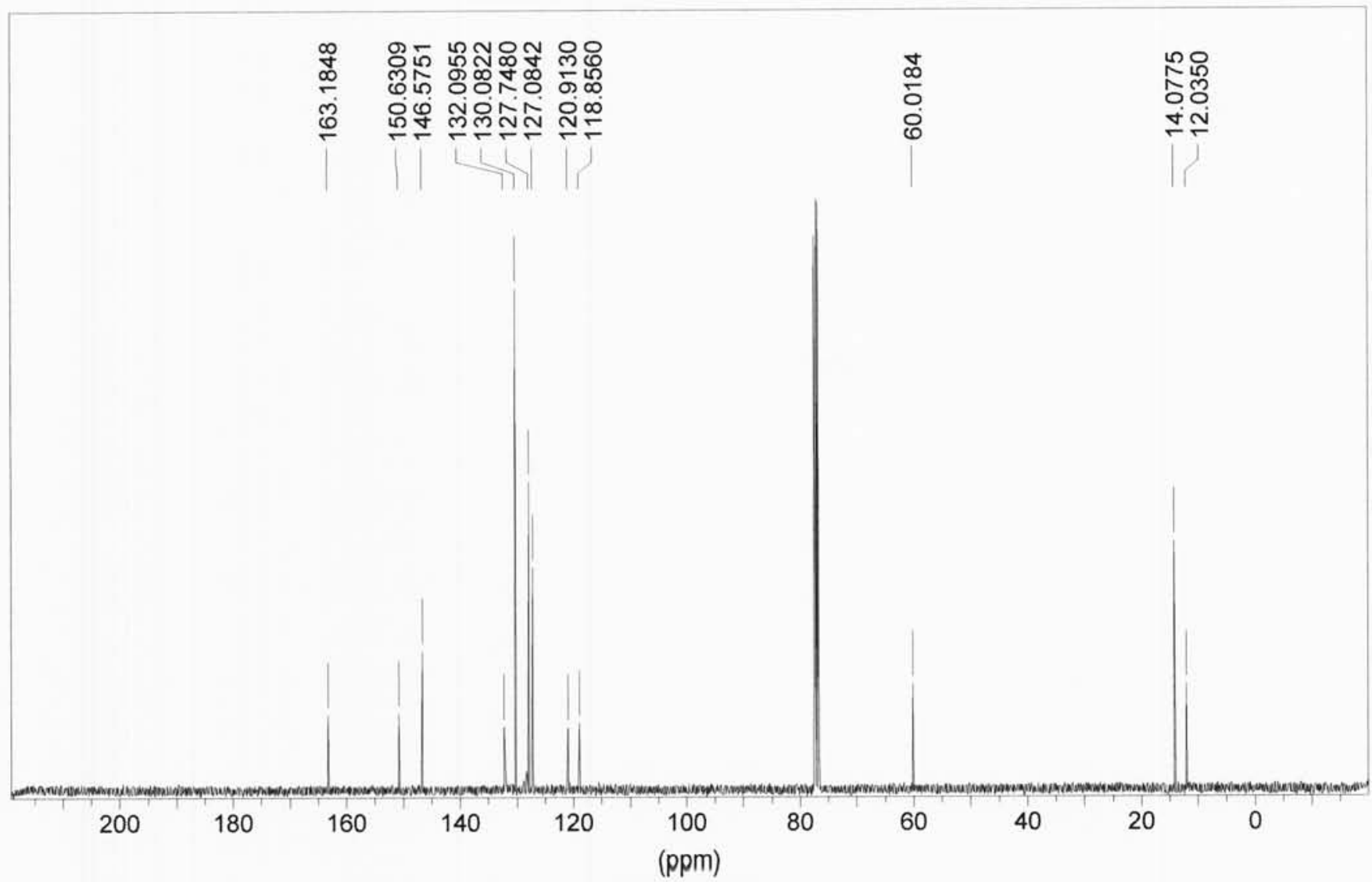



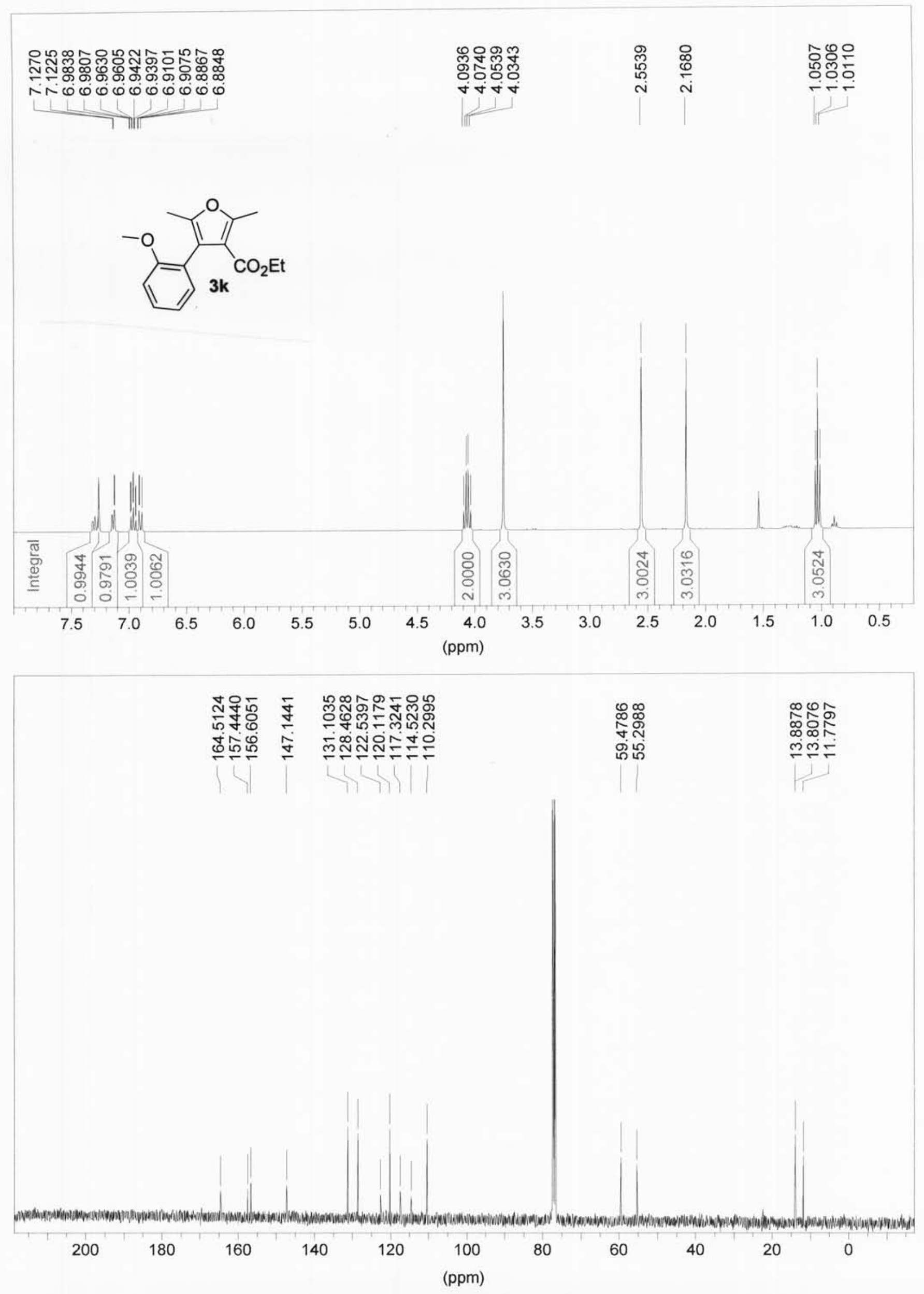

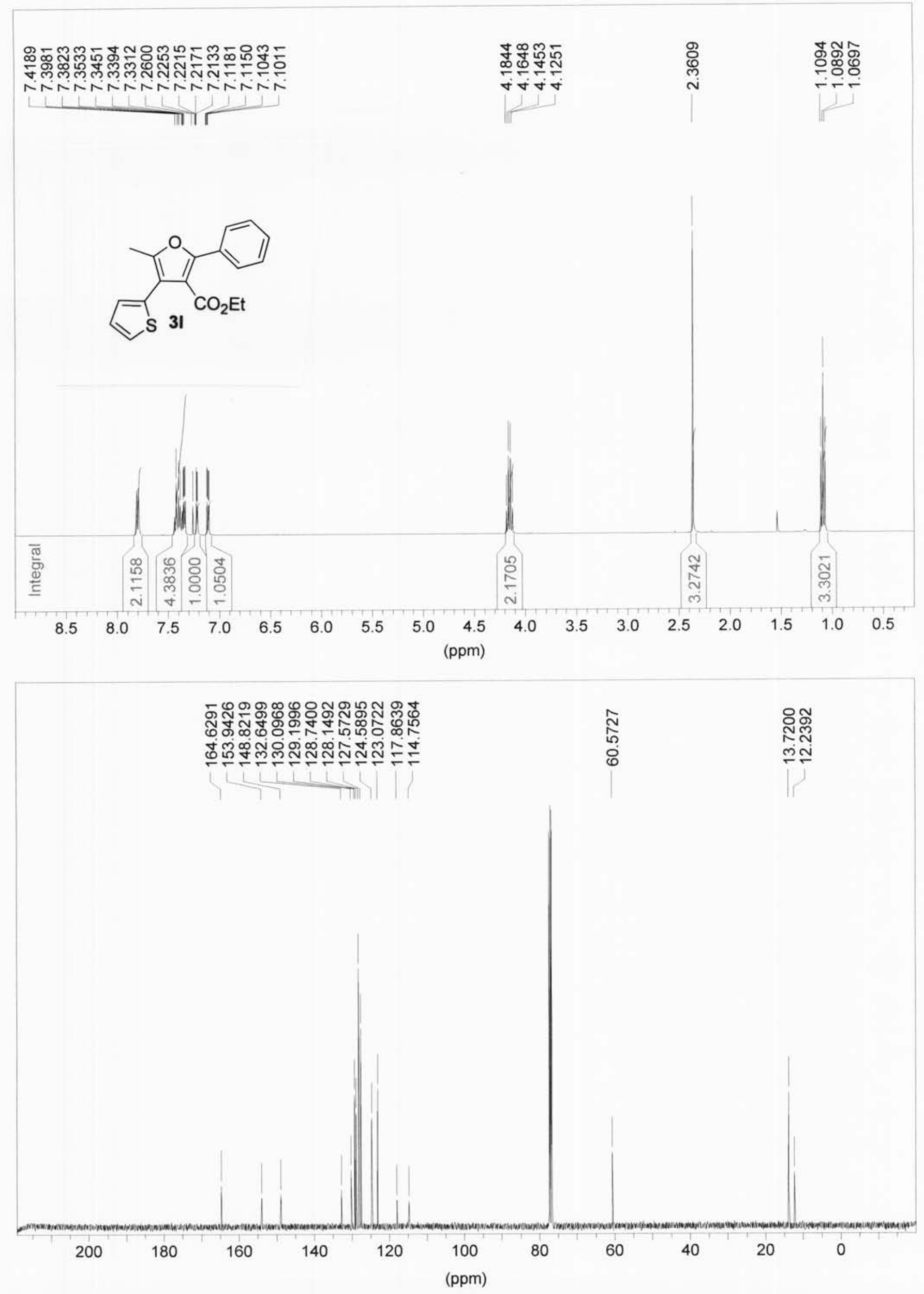

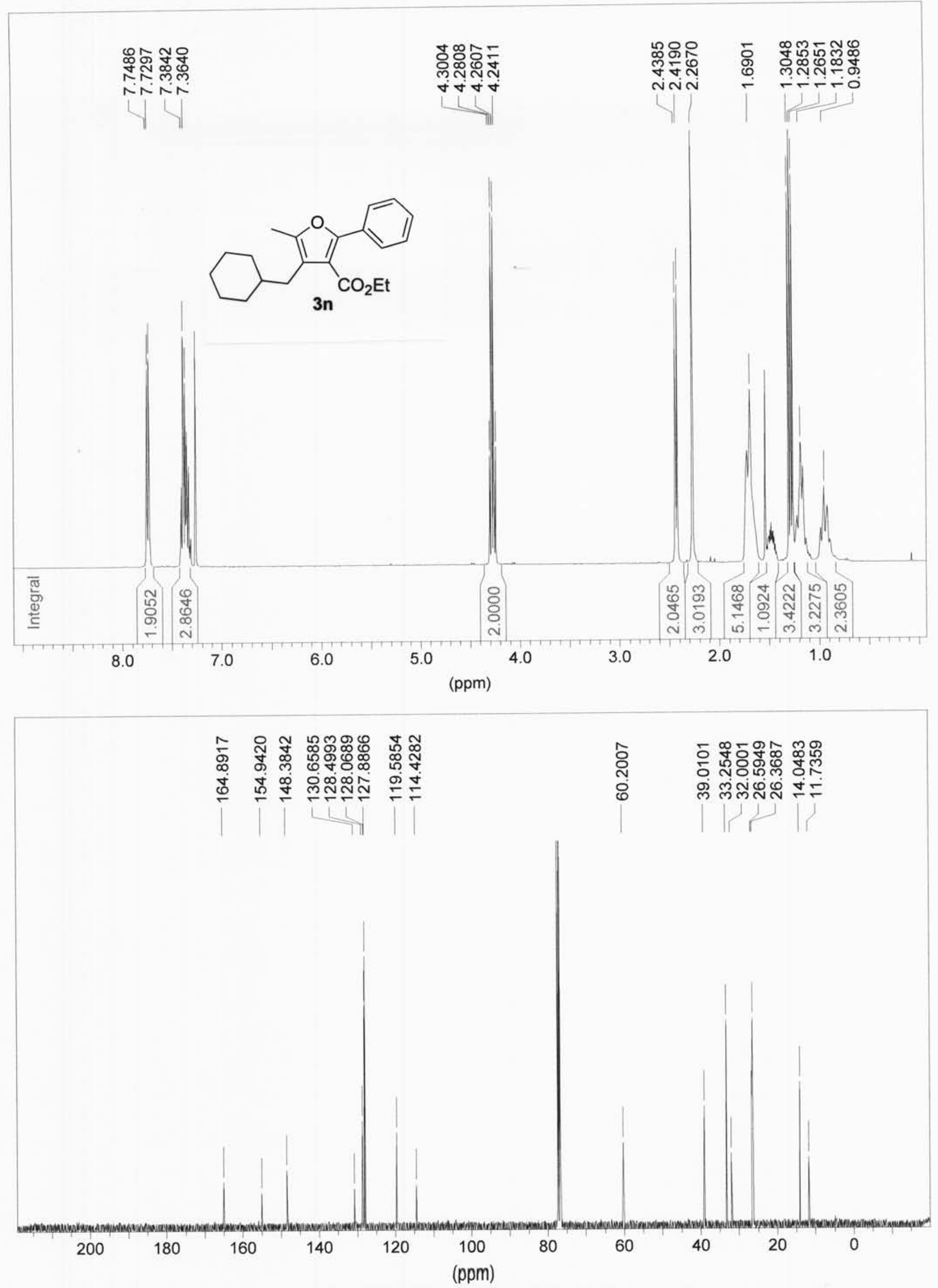

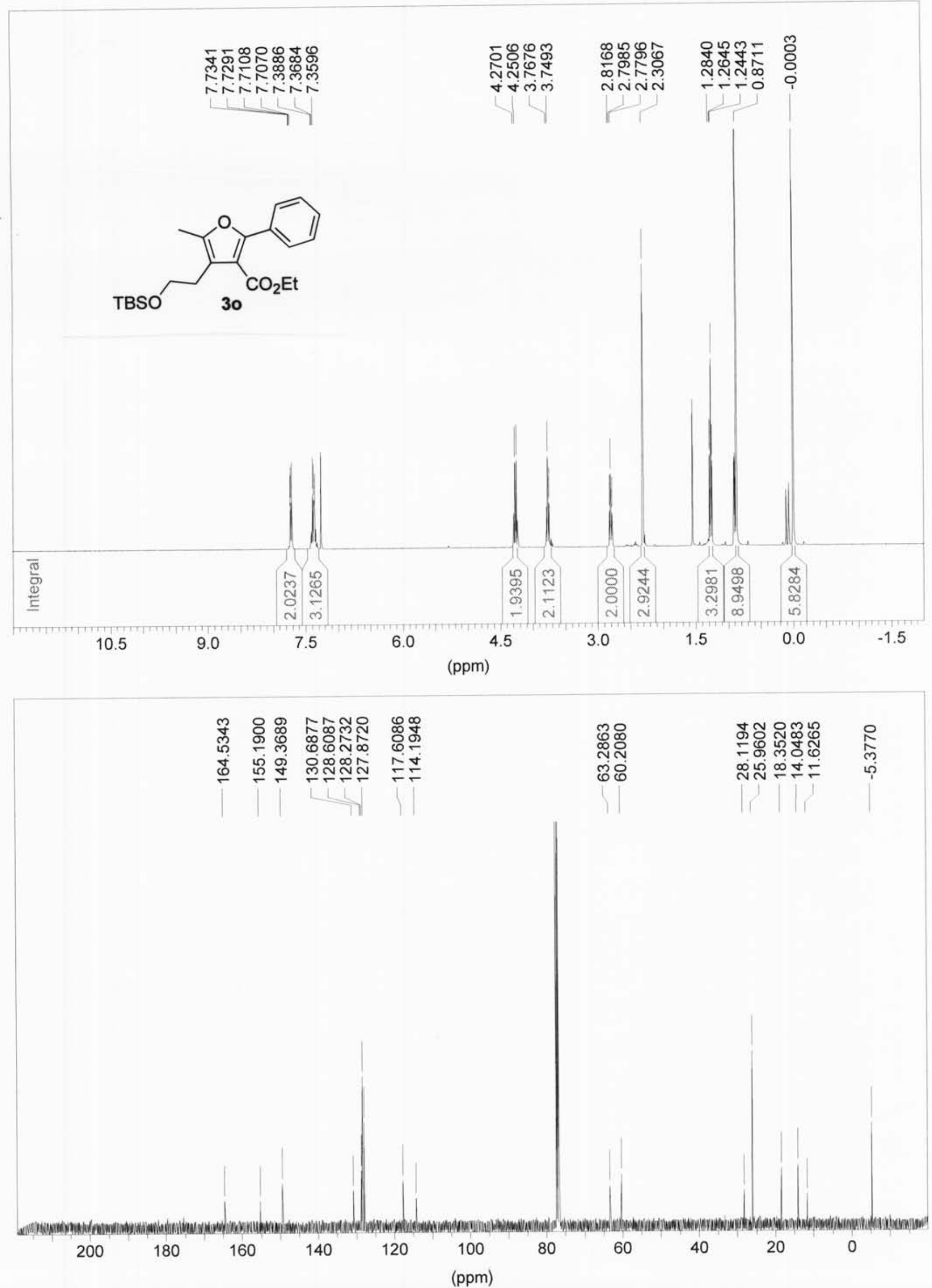

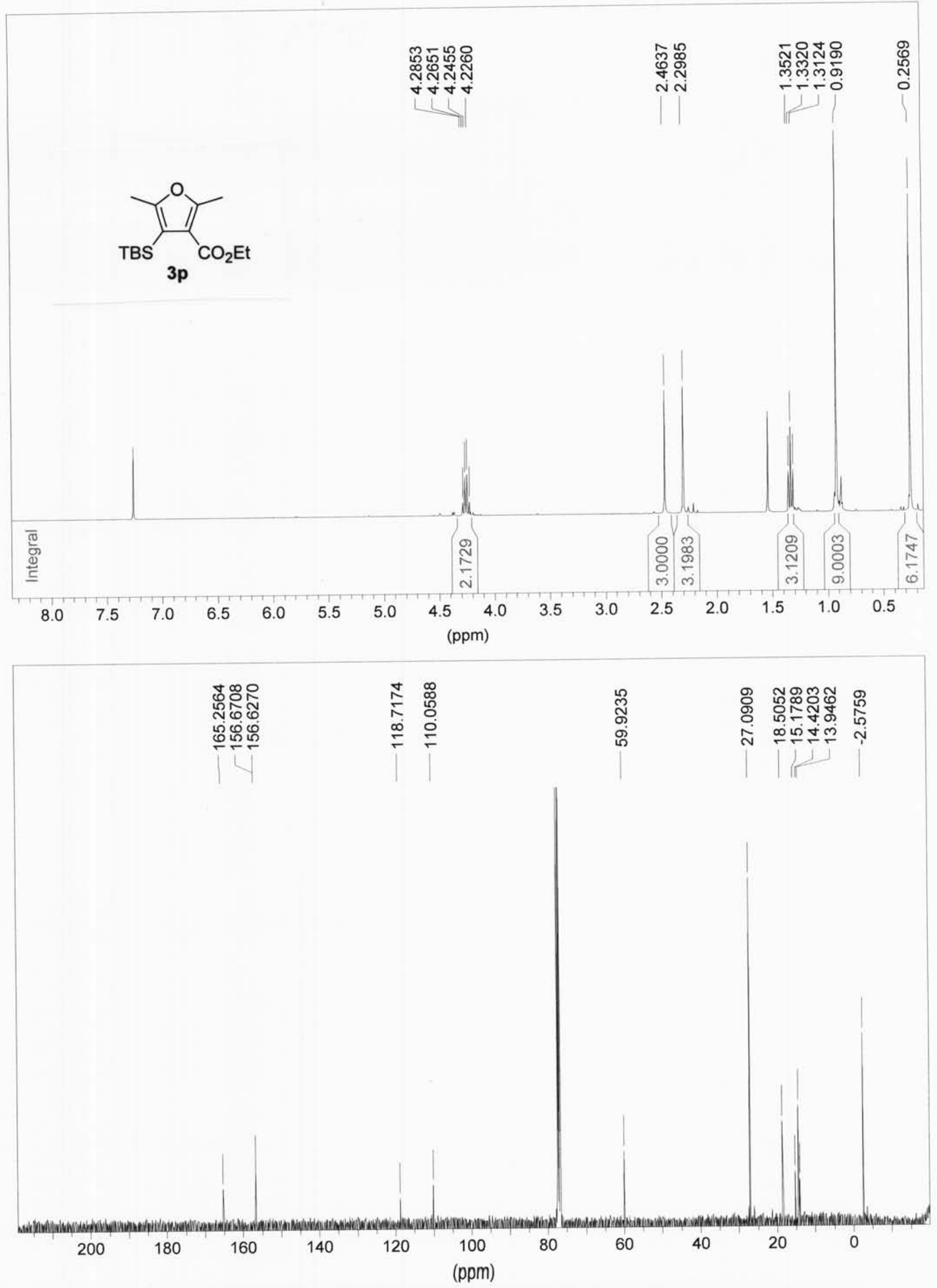

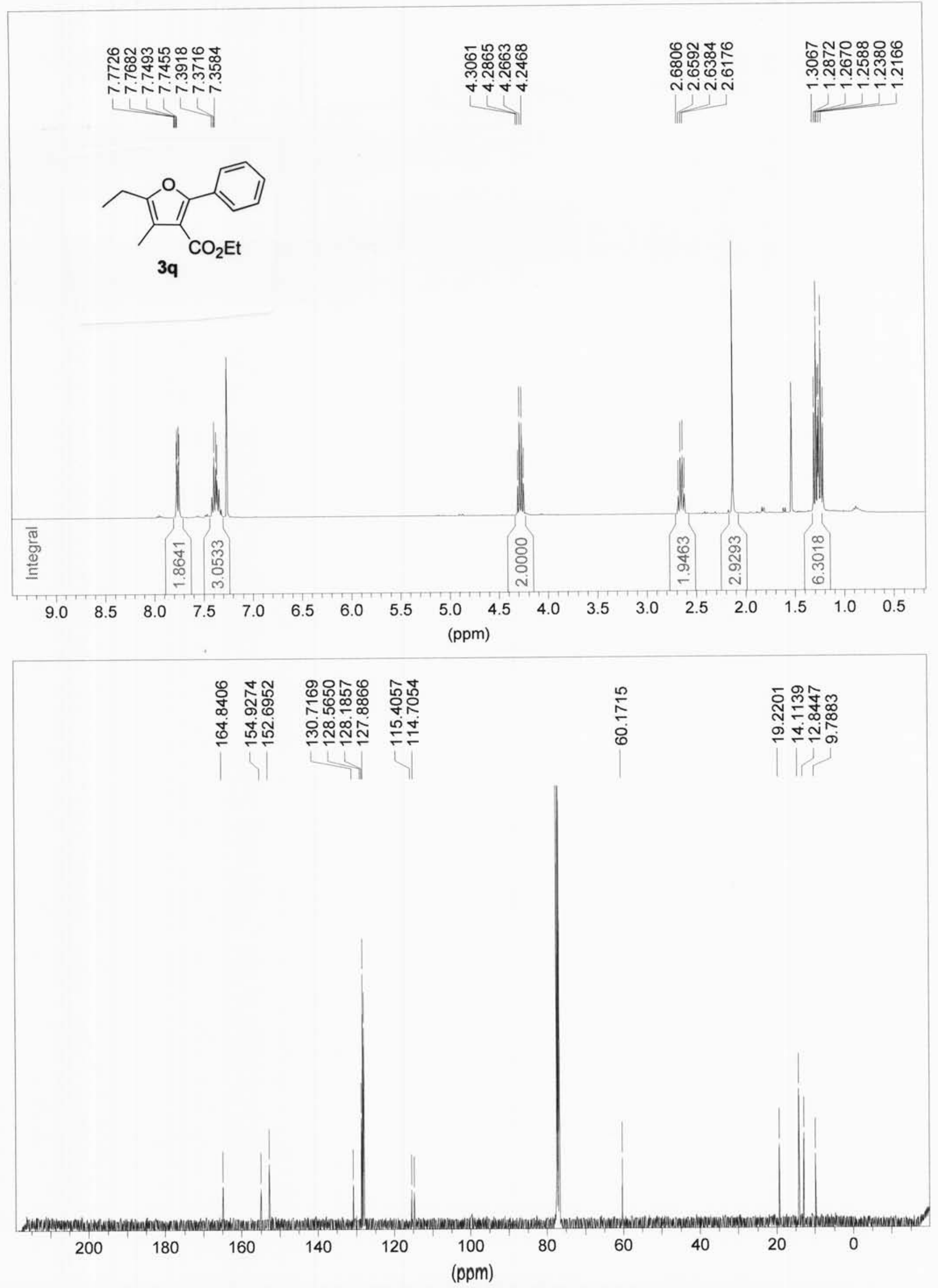